\title{
A New Approach to Modeling Morphogenesis Using Control Theory
}

\author{
Monique Chyba*, Marcelo Hissakiti Kobayashi ${ }^{\dagger}$, Youngsu \\ Cho Kwon, Frederic Mercier ${ }^{\ddagger}$, John Cuyler Rader*, Aaron \\ Tamura-Sato*
}

University of Hawai'i at Manoa Honolulu, HI 96822, USA

E-mail address: mchyba@math.hawaii.edu, marcelok@hawaii.edu, youngsu@hawaii.edu, fmercier@pbrc.hawaii.edu, jrader@hawaii.edu, aaronts@hawaii.edu

\section{Giulio Telleschi}

University di Pisa Dipartimento di Sistemi Elettrici E Automazione

Largo Lucio Lazzarino 56122 Pisa, Italy

E-mail address: giulio@eventipisani.it

\begin{abstract}
It has been proposed that biological structures termed fractones may govern morphogenic events of cells; that is, fractones may dictate when a cell undergoes mitosis by capturing and concentrating certain chemical growth factors created by cells in their immediate vicinity. Based on this hypothesis, we present a model of cellular growth that incorporates these fractones, freely-diffusing growth factor, their interaction with each other, and their effect on cellular mitosis. The question of how complex biological cell structures arise from single cells during development can now be posed in terms of a mathematical control problem in which the activation and deactivation of fractones determines how a cellular mass forms. Stated in this fashion, several new questions in the field of control theory emerge. We present this new class of problems, as well as an initial analysis of some of these questions. Also, we indicate an extension of the proposed control method to layout optimization.
\end{abstract}

* Department of Mathematics 2565 McCarthy Mall - Keller Hall 311. The research was partially supported by NSF grants: Division of Graduate Education \# 0841223, and Division of Undergraduate Education \# 0634624.

${ }^{\dagger}$ Department of Mechanical Engineering 2540 Dole Street - Holmes Hall 302. The second author was partially supported by an AFOSR grant FA9550- 10-1-0036 (monitored by Dr. David Stargel).

${ }^{\ddagger}$ Pacific Biomedical Research Center, Biomed T401 1960 East-West Road. 


\section{Introduction}

All vertebrate animals, including humans, produce new neurons and glia (the two primary specialized cell types of the brain) throughout life. Neurons and glia derive from neural stem cells, which reside, proliferate, and differentiate in specialized zones termed niches. Neural stem cells proliferate extensively during development and progressively generate the brain, a phenomenon named neurulation, or brain morphogenesis. Interestingly, neural stem cells exist and continue to generate neurons and glial cells after birth and throughout adulthood in very restricted niches, primarily the walls of the lateral ventricle (see Fig. 1).

What are the mechanisms that control neural stem cell proliferation and differentiation? Neural stem cells and their progeny respond to growth factors, endogenous signaling molecules that circulate in the extracellular milieu (in between cells). Growth factors regulate stem cell proliferation, differentiation, and migration [5]. It is not known how growth factors are selected from the extracellular milieu and dispatched within the stem cell niches to control proliferation, differentiation, and migration, and, ultimately, the architecture of the forming brain (during morphogenesis) or the plasticity (structural changes and maintenance) of the adult brain.

F. Mercier and colleagues have characterized extracellular matrix structures, named fractones, in the neural stem cell niches of the adult and developing brain $[16,13,8]$. In these niches, fractones directly contact neural stem cells and their immediate progeny [16]. Investigation of the function of fractones revealed that fractones capture growth factors from the extracellular milieu via heparan sulfates, the primary components of fractones [13]. Moreover, fractone-heparan sulfates activate growth factors at the surface of neural stem cells [9]. Since the majority of growth factors depend on heparan sulfates to exert their biological signal, fractones may represent the stem cell niche structures that capture and process a multitude of growth factors to orderly control stem cell proliferation, differentiation, and migration.

Figure 1A shows fractones aligned along the walls of the lateral ventricle, i.e. the neural stem cell niche in the adult brain. Neural stem cells proliferate next to fractones (arrows). An individual fractone is a complex structure in which processes of several stem and progenitor cells are inserted (see Fig. 1C). Transmission electron microscopy allowed us to see the detailed structure of a fractone (see Fig. 1C) [16]. Fractones also exist during development, and are associated with proliferating neural stem cells (see Fig. 2).

Inspired by these biological discoveries, we have developed a mathematical model predicting cellular proliferation from the spatial distribution of 


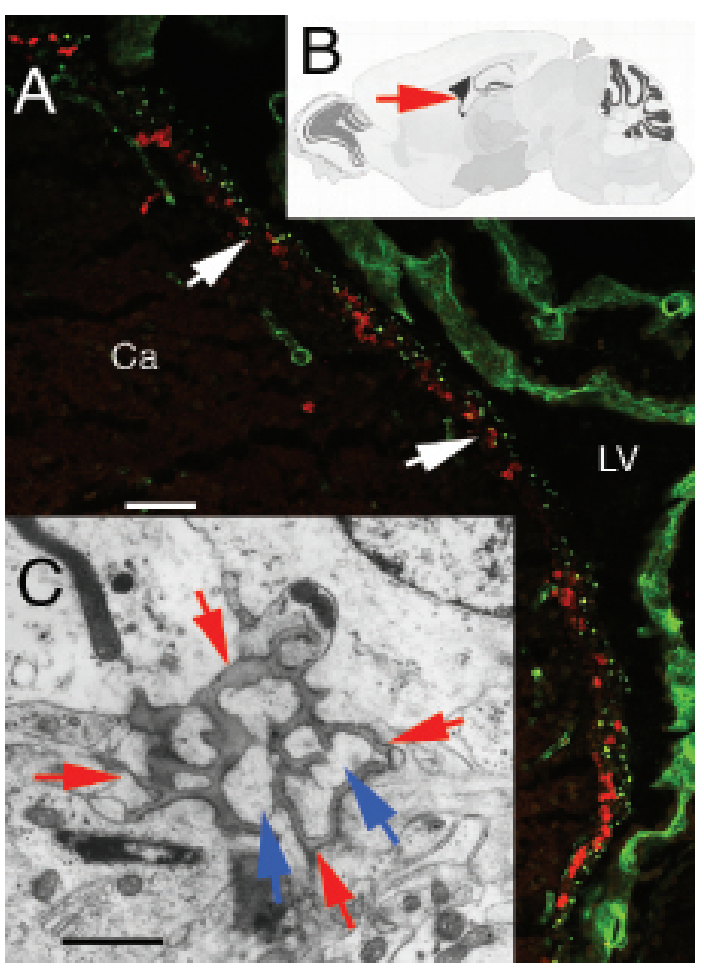

Figure 1: Fractones are extracellular matrix structures associated with proliferating cells in the neurogenic zone (neural stem cell niche) of the adult mammalian brain. A. Vizualization of fractones (green, puncta, arrows) by confocal laser scanning microscopy (Pascal Zeiss) in the primary neurogenic zone of the adult mouse brain, i.e. the wall of the lateral ventricle $(L V)$ at the surface of the caudate nucleus (Ca). Each green puncta is an individual fractone. To be detected, fractones were immunolabeled for their heparan sulfate components (using the antibody 10E4, Seikagaku, Japan) and this antibody visualized by secondary antibodies conjugated to AlexaFluor 488 (green fluorophore) [13]. The red puncta indicate proliferating neural stem cells and progenitor cells immunolabeled for the mitotic marker bromodeoxyuridine. Stem cells and their progeny proliferate next to fractones (arrows). B. Location of the confocal image A (arrow) in a schematic representation of the mouse brain (cut in the sagittal plane). $\boldsymbol{C}$. Visualization of an individual fractone by transmission electron microscopy (dark-grey structure indicated by the four red arrows. The processes of neural stem cells and of their progeny, which appear light-grey (blue arrows) are inserted into the folds of the fractone. Scale bars. A: $50 \mu \mathrm{m} ; C: 1 \mu \mathrm{m}$.

fractones. We have modeled this biological process as a control system, the control depicting the spatial distribution of the active fractones. This is a 
novel approach with respect to the most commonly reaction-diffusion (RD) models seen in the literature on morphogenesis; however, this approach is not that surprising. Indeed, the usage of control theory is instrumental to overcome many challenges faced by researchers to design systems with a very high degree of complexity and interaction with the environment
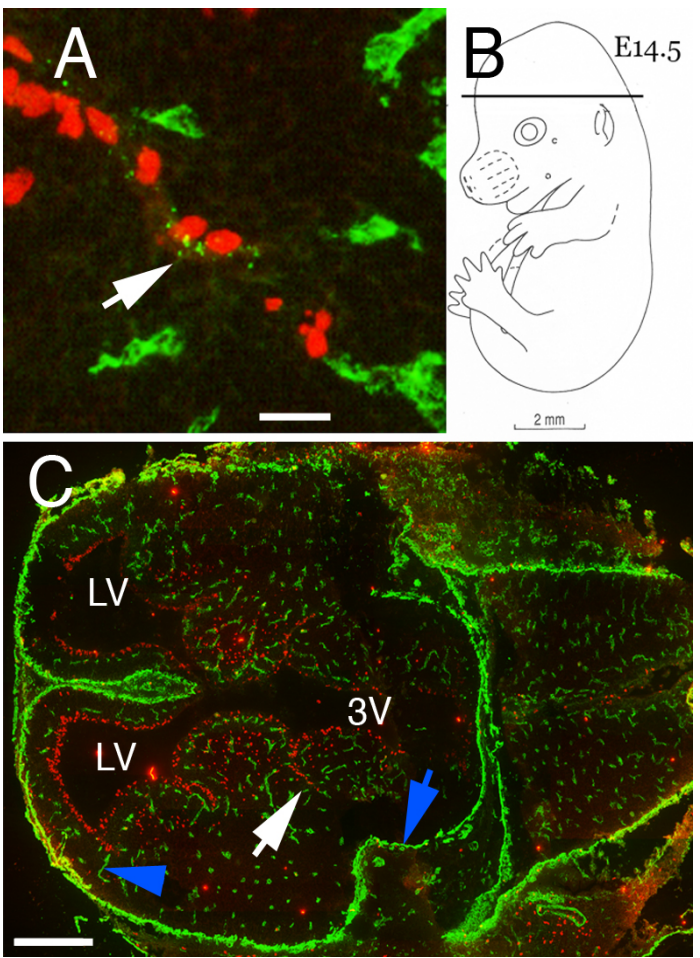

Figure 2: Fractones are associated with proliferating cells during the development of the mammalian brain. A. Fractones visualized as green puncta by immunofluorescence microscopy (DMIL Leica) in the brain of E14.5 embryos after immunolabeling for laminin (arrow). Proliferating cells (immunostained for the mitotic marker phosphrylated histone-3) are associated with fractones at the interface between the forming striatum and thalamus. $\boldsymbol{B}$. Schematic representation of an E14.5 embryo showing the plane and the location of the head sections from which originate the images $A$ and $C$. C. Low magnification of the whole head immunolabaled for laminin and phosphorylated histone-3. The microscopic field shown in the image $A$ is indicated in the low magnification image (arrow). Note that the proliferating neural stem cells are primarily located along the ventricle walls ( $L V$ : lateral ventricle and $3 \mathrm{~V}$ : third ventricle). The green lines indicate the meninges (blue arrow) and developing blood (blue arrowhead). Scale bars. A: $30 \mu \mathrm{m} ; \mathrm{B}: 0.5 \mathrm{~mm}$. 
$[6,7,19]$. Examples of the applicability of control theory in physical and biological systems are numerous [24, 25].

Due to the specific nature of morphogenesis and, in particular, cellular proliferation, our innovative model opens an entirely new area of research in control theory. As a result, new methods have to be developed to analyze biological systems from the perspective of control theory. This will advance the field of control theory by considering this new class of problems and by providing insight toward the development of innovative ideas and methods to solve these types of problems. In this paper, we present our initial analysis of this new type of control problem and some preliminary results, as well as an application of our model in the problem of layout optimization found in the fields of structural and mechanical engineering.

\section{Mathematical Model}

Our approach to model morphogenesis differs drastically from the classical R-D model. Our attempt to depict this biological process using contol theory is directly motivated by the hypothesis that fractones govern the activation of GFs to trigger cell proliferation.

2.1. Control Systems. In the literature, a control system is described by a differential equation

$$
\dot{x}(t)=f(x(t), u(t)), \quad x(t) \in M
$$

where $M$ is a $n$-dimensional manifold, and $u:[0, T] \rightarrow U, U \subset \mathbb{R}^{m}$, is the control. We assume here $f$ to be analytic. Examples of such systems includes guidance control, automation, artificial intelligence, and traffic optimization. In our case, however, the space in which the system exists is continuously evolving with time; that is, as time increases, the dimension of the space is also increasing.

2.2. Cell, Diffusion, and Fractone Spaces. Before deriving the actual dynamical model, we need to introduce the main topological spaces of interest for our model.

DEFINITION 1. The ambient space in which the morphogenic events take place is assumed to be a compact subset of $\mathbb{R}^{2}$ and, for simplicity, in this paper we assume the ambient space is fixed. We denote by $A$ a discretization of the ambient space (using for instance discretization by dilatation or Hausdorff discretization, see [22]). So, in the sequel, A is identified to a subset of $\mathbb{Z}^{2}$. The precision of the discretization is initially set by the user but eventually will be determined by the experimental biological maps. To avoid any confusion with the biological cells, in the rest of this paper, we 
call a cell of our discretization a unit and we identify each unit to an ordered pair of integer $(i, j)$. The origin unit of our discretization is chosen arbitrarily and will be identified to $(0,0)$.

The relevant components for the biological process under consideration are: the cells, the space in which the growth factors diffuse, and the fractones.

DEFINITION 2.

(i) We denote by Cell( $t)$ the configuration of cells in the ambient space at a given time $t$, and we call it the cell space. It is a closed subset of the ambient space and is identified in the sequel to a subset of $A$ (a discussion on this identification is included in Remark 1).

(ii) The diffusion space at time $t$, denoted by Diff $(t)$, represents the space in which growth factors are diffusing. It is the complement of the cell space in the ambient space and its discretization is identified to $A \backslash \operatorname{Cell}(t)$. At each time $t$, the diffusion space is split into two components, the free diffusion space Free $(t)$ where the growth factors diffuse freely, and the fractone space, Fract $(t)$ where the diffusion is perturbed. In this paper a fractone is identified to one unit of our discretization.

(iii) The data of Cell $(t)$, Free $(t)$ and Fract $(t)$ forms what we call the Configuration space at time $t$, and we denote it by $\operatorname{Conf}(t)$.

Note that $A=\operatorname{Cell}(t) \cup \operatorname{Diff}(t)$ and $\operatorname{Diff}(t)=\operatorname{Fract}(t) \cup \operatorname{Free}(t)$.

Definition 3. Let $S(t)$ be one of the spaces defined above. We define the dimension of the space $S$ at time $t$ as the number of indices $(i, j)$ such that $(i, j) \in S(t)$ where $S(t)$ has been identified to its discretization.

Topologically, we can interpret the above definitions as follows: we can visualize the configuration space at a given time $t$ as a compact subset of $\mathbb{R}^{2}$ with holes depicted by the cells. On a given discretization of this topological space (varying with time), we will model the diffusion of growth factors (which is perturbed at the location of a fractone). Finally, we will incorporate into our model the mechanisms that allows duplication of cells.

Remark 1. From a purely theoretical point of view, there are many ways that we can represent the cells in the ambient space. Indeed, we can make the assumption that they are all circular and have the same dimension or that their shape differs in size and form. Notice that, from a practical point of view, the shape and size of the cells of the initial configurations will be given by the experimental map and its discretization. To write the dynamic model of our biological process we only assume that the size and shape of all cells are identical as well as the fact that the cells are vertically and horizontally aligned. The assumptions made for the simulations presented in this paper are clearly explained in Example 1. Also, in this paper, we make the assumption that a fractone is one unit of our discretization. In 
future work, we plan to relax this assumption to allow more complex forms for the fractones.

Example 1. Consider the ambient space shown in Figure 3 at time $t=0$, where individual cells are represented by large circles, and fractones are represented by small circles. The cells are drawn spaced apart so that individual cells are distinguishable and fractones are clearly visible, but in reality the cells may be pushed up against one another. In Figure 4, we

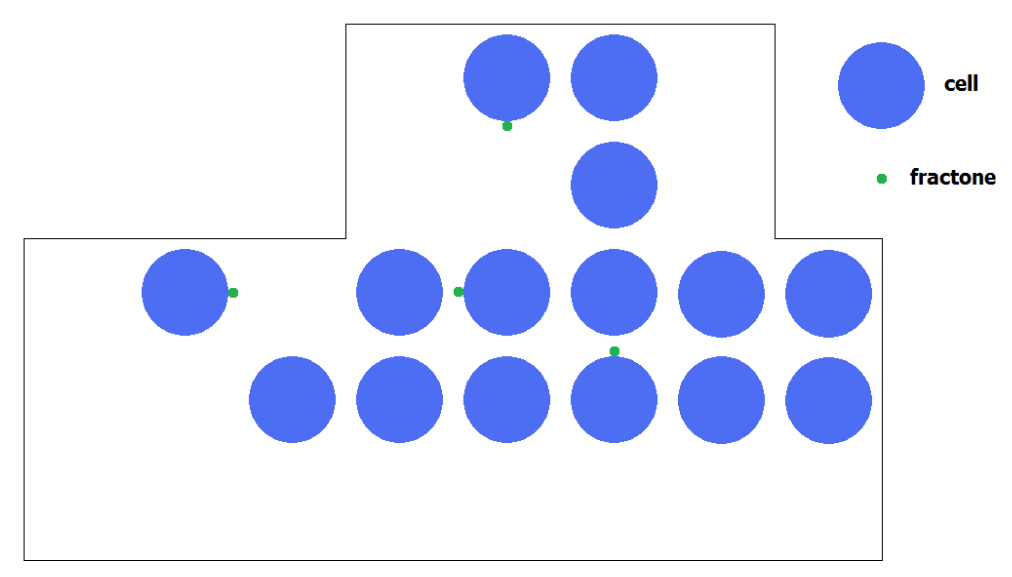

Figure 3: An ambient space with 14 cells and 4 fractones. Note the assumption of uniform cell size, uniform cell shape, and alignment of cells.

discretize to the ambient space (but not the cells). We assume that the space between cells accounts for $33 \%$ of the total space occupied by the cell. Thus, in the figure, we choose a precision such that each large circle is inscribed in a $9 \times 9$ square with a 1 unit border. This $11 \times 11$ square represents one cell with associated spacing. Fractones fit within a single unit. Then $A=\{(i, j) \in \mathbb{Z} \times \mathbb{Z} \mid 0 \leq i \leq 87,0 \leq j \leq 32\} \bigcup\{(i, j) \in$ $\mathbb{Z} \times \mathbb{Z} \mid 33 \leq i \leq 76,33 \leq j \leq 54\}$. We note that the location of the origin, $(0,0)$, was an arbitrary choice. Figure 5(a) shows the full discretization. Each cell is represented by a $9 \times 9$ square with a 1 unit border. The border is not considered part of the cell space, but is part of the diffusion space. This 2 unit wide "channel" between neighboring cells allows growth factor to diffuse between cells. Each fractone is represented by a single unit. It 


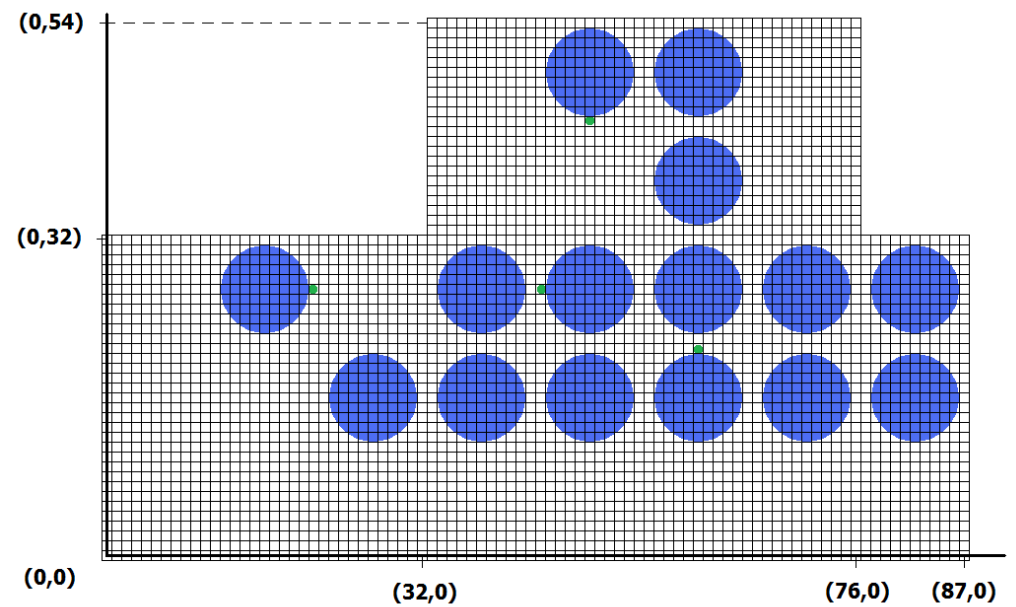

Figure 4: The ambient space with origin chosen to be on the bottom left. Each large circle representing a cell is 9 units in diameter. Each fractone is 1 unit in diameter. There is a 2 unit space between cells.

follows that the cell space in the example is given by:

$$
\begin{aligned}
& \operatorname{Cell}(0)=\{(i, j) \mid i \in\{23,24, \ldots, 86\} \backslash\{32,33,43,44,54,55,65,66,76,77\}, \\
& j \in\{12,13, \ldots, 20\}\} \bigcup\{(i, j) \mid i \in\{12,13, \ldots, 86\} \backslash\{21,22, \ldots, 33,43,44, \\
& 54,55,65,66,76,77\}, j \in\{23,24, \ldots, 31\}\} \bigcup\{(i, j) \mid i \in\{56,57, \ldots, 64\}, \\
& j \in\{34,35, \ldots, 42\}\} \bigcup\{(i, j) \mid i \in\{45,46, \ldots, 64\} \backslash\{54,55\}, \\
& j \in\{45,46, \ldots, 53\}\}
\end{aligned}
$$

Moreover, we have:

$$
\begin{aligned}
\operatorname{Fract}(0) & =\{(21,27),(44,27),(49,44),(60,21)\} \\
\operatorname{Free}(0) & =A \backslash(\operatorname{Cell}(0) \cup \operatorname{Fract}(0)) \\
\operatorname{Diff}(0) & =\operatorname{Fract}(0) \cup \operatorname{Free}(0) .
\end{aligned}
$$

Previous simulations where cell spacing was assumed to be only $20 \%$ of the total space occupied by the cell had 1 unit wide channels. In these simulations, the diffusion process was occuring much to slow compared versus reality. 


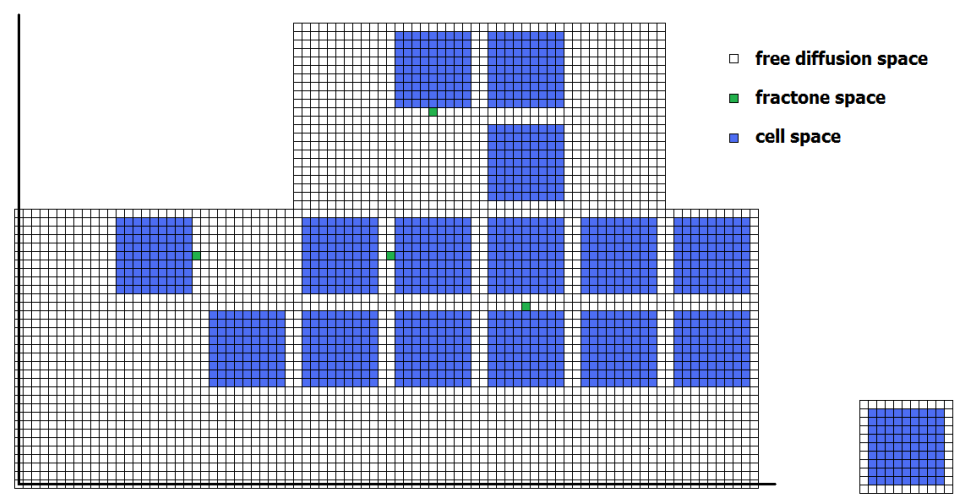

(a)

(b)

Figure 5: (a) The discretization of the original ambient space. Note that the free diffusion space and the fractone space together form the diffusion space. (b) A "cell": a $9 \times 9$ square in the cell space with a 1 unit border in the diffusion space

In our proposed model, the morphogenic events will be governed by a control system defined on a state space. In this paper, we assume only one growth factor is diffusing in the space. The state space is defined at each time $t$ as the concentration of growth factor in each unit of our discretization of the diffusion space $\operatorname{Diff}(t)$. We denote the state space by $M(t)$. More precisely, since there is a one-to-one correspondence between units and ordered pairs of integer, we have:

Definition 4. Let $(i, j) \in \operatorname{Diff}(t)$. At each time $t$, we introduce the concentration of growth factor in unit $(i, j)$ that we denote by $X_{i, j}(t)$. The state space $M(t)$ at time $t$ is then $M(t)=\mathbb{R}_{\geq 0}^{\operatorname{dim}(\operatorname{Diff}(t))}$.

As will be seen later, the rate of change in the concentration of growth factor is described using classical diffusion equations.

Remark 2. Due to the morphogenic nature of the biological process under study, the state space is constantly evolving as well as the configuration space. This distinguishes in a very non-trivial way our problem from the traditional problems in engineering or physics whose systems are usually defined on a static configuration space.

2.3. Moded for the Perturbed Diffusion of Growth Factors. For simplicity, we assume the diffusion of a unique type of growth factor and 
equal sensitivity of the fractones with respect to that growth factor. However, our model is developed such that expanding to several types of growth factors and varying fractone sensitivity to respective growth factors can be added in a straightforward way.

Our assumption that fractones act like captors implies that the diffusion of growth factor in the extra-cellular space is being perturbed from a regular diffusion process. We model the diffusion process as an affine control system defined at each $t$ on the state space $M(t)$ :

$$
\dot{X}(t)=F^{0}(X(t))+\sum_{(i, j) \in \operatorname{Diff}(t)} F^{(i, j)}(X(t)) \cdot u_{(i, j)}(t), \quad X(t) \in M(t) .
$$

Here, the drift vector field $F^{0}(x(t))$ represents pure diffusion as if no fractones exist, the control vector fields $F^{(i, j)}(X(t))$ reflect the perturbation in the diffusion process when fractones are present, and $u($.$) is called the$ control. Notice that, for the diffusion process, the cells play the role of obstacles. This is captured in equation (2) with the sum acting on indices $(i+k, j+l)$ that belongs to the diffusion space only, so if a unit of our discretization belongs to a biological cell, then the diffusion is prevented to and from that unit.

In order to accurately describe these mechanisms, let us first introduce $\Delta=\{(0,1),(0,-1),(1,0),(-1,0)\}$, and $\nu$ the diffusion parameter associated to the considered growth factor. The drift vector field is given by:

$$
F^{0}(X(t))=\nu \cdot \sum_{\substack{(k, l) \in \Delta \\(i+k, j+l) \in \operatorname{Diff}(t)}}\left(X_{i+k, j+l}(t)-X_{i, j}(t)\right) .
$$

The perturbation of the pure diffusion due to the fractones existence is modeled as follows. As mentioned before, a fractone is identified to a single unit $(i, j)$ of our discretization. The hypothesis is that every active fractone stores the quantity of growth factors that it captures, and that this quantity becomes unavailable to the diffusion process. Moreover, a key element in our hypothesis is that the spatial distribution of fractone varies through the sequence of morphogenic events. The role of the function $u($.$) introduced$ in equation (2) is precisely to control the location and activation of the fractones. Mathematically, we assume that a passive fractone exists in every unit of the free space which biologically corresponds to the situation when either there is no fractone or the fractone is being produced but is not yet active. Once a fractone becomes active, the unit shifts from the free space into the fractone space. This leads to the following definition:

Definition 5. To each unit $(i, j) \in$ Free $(t)$, we associate what we call a passive fractone. A passive fractone does not perturb the diffusion process. An active fractone at time $t$ is defined as a unit of our discretization that 
belongs to the set $\operatorname{Fract}(t)$. An active fractone is one that acts as a captor for the diffusion process.

Let $(i, j) \in \operatorname{Diff}(t)$. The control function is then defined as:

$$
u_{(i, j)}(t)=\left\{\begin{array}{ll}
0 & \text { if }(i, j) \in \operatorname{Free}(t) \\
1 & \text { if }(i, j) \in \operatorname{Fract}(t)
\end{array} .\right.
$$

DeFinition 6. Let $T$ be the duration of the cascade of morphogenic events under study. An admissible control is a measurable function $u:[0, T] \rightarrow$ $\{0,1\}^{\operatorname{dim}(\operatorname{Diff}(t))}$ that satisfies equation (4).

From our definitions, we now have that, when the fractone identified to unit $(i, j) \in \operatorname{Diff}(t)$ becomes active at time $t$, the component $u_{i, j}(t)$ of the control is turned on to 1 . Then, the active fractone stores the current quantity of growth factors available in unit $(i, j)$ and acts as captor for the diffusion process. In other words, diffusion from a unit $(i, j) \in \operatorname{Fract}(t)$ to its neighbors is prevented. More precisely, under the assumption that $(i, j) \in \operatorname{Fract}(t)$, we have:

$$
\begin{gathered}
F_{i, j}^{(i, j)}(X(t))=\nu \cdot \sum_{\substack{(k, l) \in \Delta \\
(i+k, j+l) \in \operatorname{Diff}(t)}} X_{i, j}(t) \\
F_{i+k, j+l}^{(i, j)}(X(t))=-\nu \cdot X_{i, j}(t) \text { for } \begin{array}{c}
(k, l) \in \Delta, \\
(i+k, j+l) \in \operatorname{Diff}(t)
\end{array}
\end{gathered}
$$

Those equations reflect the fact that the quantity of growth factor in an active fractone becomes invisible to the diffusion process. Once the stored quantity reaches a given threshold, the fractone signals to the cells that mitosis can occur.

Example 2. Figures 6-7 illustrate the perturbed diffusion process on the configuration space introduced in the previous examples. We represent three snapshots of a diffusion process resulting from two single sources of growth factor as it can be seen in the first picture of Figure 6. The third dimension represents the quantity of GF in the corresponding unit. In red are the cells, light blue represents the free space, the dark blue spaces do not belong to the ambient space (they represent obstacles for the diffusion and mitosis) and, as before, in green we represent the active fractones.

2.4. Mitosis. As mentioned in the introduction, growth factors are regularly produced by the biological cells and then are diffusing freely in the available extra-cellular space. When the growth factor is significantly close to an active fractone, said fractone captures and concentrates the growth factor. Once the concentration of growth factor reaches a significant value, the fractone gives the order to its associated cell to undergo mitosis. In 
reality, the time for a cell to undergo mitosis is approximately four hours. However, by the time mitosis actually occurs, the fractone may have relocated. What is interesting is that the previous location of a fractone has been shown to be the location of new cells after the next morphogenic event. Due to this correlation, it is clear that the spatial distribution of fractones dictates the location of future morphogenic events, hence the fractones are the obvious choice to represent the controls in our system. One may argue that the reality does not match the model in that there is a time lapse in which the fractones may or may not move, and the cell undergoes mitosis. To alleviate this problem, the model is so that mitosis occurs instantaneously once an order has been issued by a fractone, and that fractone movement is also instantaneous in that we associate every avaiable unit in Free $(t)$ with a fractone, and that "moving" a fractone is equivalent to changing the control from 1 to 0 in one location (making this fractone inactive) and vice-versa in another location (making this fractone active). To equivalently describe this process mathematically, we state that the spatial distribution of the fractones and the concentrations of freelydiffusing growth factors dictate the location and appearance of holes (i.e. cells) in the configuration space.

Now that mitosis is occuring, a natural question arises: when a cell undergoes mitosis, how does the existing mass of cells deform? The deformation of the mass of cells undergoing morphogenic events is extremely complex. Indeed, it involves many different criteria to take into account as well as forces to optimize. Our goal in this paper is to state and start analyzing some control problems formulated in a new setting rather than to produce the most accurate simulation of the biological process which would render such a complex system that an analytic study could not be conducted. Therefore, our criterion for the deformation of the mass of cell is based on the minimization of a given distance function.

DEFINITION 7. We assume in the sequel that we have a distance function, denoted by d, defined on the set of ordered pairs of integers. More precisely, for each $a=\left(a_{1}, a_{2}\right), b=\left(b_{1}, b_{2}\right) \in \mathbb{Z} \times \mathbb{Z}$, the distance between $a$ and $b$ is well defined by the positive real number $d(a, b)$.

When mitosis occurs at a given time $t$, the configuration space $\operatorname{Conf}(t)$ undergoes a topological change. Indeed, with new cells forming, they become additional holes in the ambient space, and while the dimension of Free $(t)$ decreases, the dimension of Cell $(t)$ increases. To accomodate for the formation of new cells, Cell $(t)$ has to deform accordingly to a prescribed algorithm. Assume unit $(i, j)$ represents an active fractone and we denote by $C$ the associated cell (under our current assumptions a fractone can be linked to a single cell only). Since we assume all cells rigid and of 
equal shape, we can identify a biological cell to a single unit of our discretization. For instance, in Example 1, each cell can be identified to its middle unit denoted here by $(a, b)$ and we can write $C=(a, b)$. The deformation algorithm is defined as to preferentially deform the current mass of cells in the direction of the closest empty space in a clockwise orientation as starting from angle zero (as referenced by an axis superimposed on the center of the "mother" cell). More precisely, assume $C=(a, b)$ duplicates. The algorithm looks incrementally for the closest unit $(i, j) \in$ Free $(t)$ to $(a, b)$ (based on the chosen distance $d$ ) such that $(i, j)$ can be identified to a cell (see Example 3 for more details). Since more than one unit identified to a cell can be at the same distance from $(a, b)$, we need to use a selection algorithm. There are many ways to select among those units; it could even arbitrarily be determined by the computer. See Example 3 for the choice made for our simulations.

Example 3. This example uses the assumptions made in Examples 1 and 2. For the simulations included in this paper, we use the following deformation algorithm. Let $\alpha_{1}=\left(a_{1}, b_{1}\right), \alpha_{2}=\left(a_{2}, b_{2}\right) \in \mathbb{Z} \times \mathbb{Z}$, we consider the Euclidean distance:

$$
d_{E}\left(\alpha_{1}, \alpha_{2}\right)=\sqrt{\left|a_{1}-b_{1}\right|^{2}+\left|a_{2}-b_{2}\right|^{2}} .
$$

Our choice of distance is motivated by the assumption that the mass of cells is optimizing its shape by prioritizing compactness. As it was explained above, we assume that each cell is identified to its middle unit of its discretization. It is therefore understood that $C=(a, b)=\{(i, j) \in$ $\operatorname{Cell}(t) ; a-4 \leq i \leq a+4, b-4 \leq j \leq b+4\}$. Let $C_{i}=\left(a_{i}, b_{i}\right)$, $i=1,2$ be two biological cells. Therefore, since every cell has a unitwide boundary, then $a_{1}=a_{2} \bmod 11$ and $b_{1}=b_{2} \bmod 11$ and $d_{E}\left(C_{1}, C_{2}\right) \in$ $\left\{11 \sqrt{n^{2}+m^{2}} \mid n, m \in \mathbb{Z}\right\}$. It follows that the deformation algorithm will search for the closest units in Free $(t)$ that are at distances of the form $11 \sqrt{n^{2}+m^{2}}$ from the cell undergoing mitosis. Notice that, given a cell $C$, the closest units multiples of 11 from $C$ are at a distance 1 (i.e. $(n, m)=$ $(0,1)$ or $(1,0))$, and there are 4 of them. The next closest units of 11 are at a distance $\sqrt{2}$, and there are also 4 of them. Table 1 list some of the possible distances (divided by 11 in the table). The pattern is very clear.

(1) 12 possible units if $d_{E}=11 * r$ where $r$ is an integer that is the hypotenuse of a Pythagorean triple,

(2) 8 possible units if $d_{E}=11 * r$ where $r$ is not along a diagonal or an axis in Table 1 , or

(3) 4 possible units if $d_{E}=11 * r$ where $r$ is on a diagonal or an axis, and is not the hypotenuse of a Pythagorean triple. 


\begin{tabular}{|c||c|c|c|c|c|c|c|c|c|c|}
\hline 0 & 1 & 2 & 3 & 4 & 5 & 6 & 7 & 8 & 9 & 10 \\
\hline \hline 1 & $\sqrt{2}$ & & & & & & & & & \\
\hline 2 & $\sqrt{5}$ & $\sqrt{8}$ & & & & & & & & \\
\hline 3 & $\sqrt{10}$ & $\sqrt{13}$ & $\sqrt{18}$ & & & & & & & \\
\hline 4 & $\sqrt{17}$ & $\sqrt{20}$ & $\sqrt{25}$ & $\sqrt{32}$ & & & & & & \\
\hline 5 & $\sqrt{26}$ & $\sqrt{29}$ & $\sqrt{34}$ & $\sqrt{41}$ & $\sqrt{50}$ & & & & & \\
\hline 6 & $\sqrt{37}$ & $\sqrt{40}$ & $\sqrt{45}$ & $\sqrt{52}$ & $\sqrt{61}$ & $\sqrt{72}$ & & & & \\
\hline 7 & $\sqrt{50}$ & $\sqrt{53}$ & $\sqrt{58}$ & $\sqrt{65}$ & $\sqrt{74}$ & $\sqrt{85}$ & $\sqrt{98}$ & & & \\
\hline 8 & $\sqrt{65}$ & $\sqrt{68}$ & $\sqrt{73}$ & $\sqrt{80}$ & $\sqrt{89}$ & $\sqrt{100}$ & $\sqrt{113}$ & $\sqrt{128}$ & & \\
\hline 9 & $\sqrt{82}$ & $\sqrt{85}$ & $\sqrt{90}$ & $\sqrt{97}$ & $\sqrt{106}$ & $\sqrt{117}$ & $\sqrt{130}$ & $\sqrt{145}$ & $\sqrt{162}$ & \\
\hline 10 & $\sqrt{101}$ & $\sqrt{104}$ & $\sqrt{109}$ & $\sqrt{116}$ & $\sqrt{125}$ & $\sqrt{136}$ & $\sqrt{149}$ & $\sqrt{164}$ & $\sqrt{181}$ & $\sqrt{200}$ \\
\hline
\end{tabular}

Table 1: Sample distance distribution for the deformation of the mass of cells as measured from the "mother" cell (located at 0). Here, only one half of one quadrant is displayed since it is symmetrical with respect to the other quadrants, and the table is symmetrical about its diagonal.

To select among several units at the same distance $d_{E}$ from the duplicating cell $C=(a, b)$, we define $i_{\ell}-a$ and $j_{\ell}-b$, for all $\ell$, where $\ell$ represents the number of possible locations at a given distance. The algorithm looks first for a unit in Free $(t)$ such that $j_{\ell}-b \leq 0$ and chooses preferentially the $\max \left\{i_{\ell}\right\}$ and its corresponding coordinate. If no such unit is found, the algorithm searches for a unit in Free $(t)$ such that $j_{\ell}-j_{0}>0$, and chooses preferentially the $\min \left\{i_{\ell}\right\}$ and its corresponding coordinate. Once the unit at the shortest distance $d_{E}$ is detected, the deformation occurs in that direction. In Figure 8, we display a sequence of morphogenic events to illustrate how our deformation algorithm works.

When a cell undergoes mitosis and the distance algorithm has chosen a position in Free $(t)$ for Cell $(t)$ to deform toward (let us refer to this closest selected unit at a distance $d_{E} \bmod 11$ as $(c, d)$ ), the growth factor present in the space must move in order to make room for the deformed mass of cells. Hence, the algorithm for redistribution of GF occurs as follows:

(1) it calculates the sum of the GF present in the space associated to a cell centered in unit $C=(c, d)$ where the mass of cells will deform toward, i.e.

$$
\sum_{k, l=-4}^{4} X_{c+k, d+l}(t) \text {. }
$$

(2) deforms $\operatorname{Cell}(t)$ such that $(c, d) \in \operatorname{Cell}(t)$. 
(3) counts the number of units in Free $(t) \cup \operatorname{Frac}(t)$ that are at a distance $d_{E} \leq 8$ from $(c, d)$.

(4) distributes $70 \%$ of the sum from (1) evenly in each unit from (3).

(5) counts the number of units in Free $(t) \cup \operatorname{Frac}(t)$ at a distance $8<$ $d_{E} \leq 11$ from $(c, d)$.

(6) distributes the remaining $30 \%$ of the sum from (1) evenly in each unit from (5).

In this way, one can see that once the new cell enters the system, the deformation of $\operatorname{Cell}(t)$ creates a "pressure wave" that distributes the GF around the space where the deformation impacts Free $(t)$. It should be noted that the distances and percentages chosen are arbitrary and are easily adjustable. In Figure 9, we represent a simulation of a sequence of morphogenic events. We display the cell's duplications as well as the diffusion of growth factors. For our simulations, we also included in the model that every biological cell in the simulations produce GF, and that the units that comprise the edge of a cell produce an amount of GF equal to 0.2 / unit / 3 seconds, that way the whole cell can be thought of as producing an amount of GF equal to 7.2 / 3 seconds (since there are 36 edge units in a $9 \times 9$ cell). Accordingly, the GF threshold for a fractone is currently set at 0.4 .

Fig. 9(a): : Initial cell space.

Fig. 9(b-c): : The initial cell produces growth factor, which diffuses and is captured by the neighboring fractone at $(23,18)$.

Fig. 9(d): : The cell undergoes mitosis. The fractone at $(23,18)$ deactivates and a fractone at $(34,18)$ activates.

Fig. 9(e-h): : Cells continue to produce growth factor, which diffuses and is captured by the fractone at $(34,18)$, triggering mitosis when concentrations are high enough.

Fig. 9(h-j): : The fractone at $(34,18)$ deactivates, and a fractone at $(34,7)$ activates until mitosis occurs

Fig. 9(k-n): : The fractone deactivates and a fractone at $(13,18)$ activates until mitosis occurs.

Fig. 9(n-0): : Finally, the fractone at $(13,18)$ deactivates, and the fractone at $(34,18)$ reactivates, prompting mitosis when growth factor concentrations are high enough.

\subsection{Problem Formulation.}

2.5.1. Biological formulation. .

Given an initial and final configuration of cells in a prescribed ambient space, determine an initial concentration of growth factors and a dynamic 
spatial distribution of fractones such that the mass of cells transforms from its initial configuration to its final configuration.

\subsubsection{Mathematical formulation. .}

Let us now rephrase this using the mathematical definitions introduced previously. To summarize, the quantity of growth factor in each unit of our discretization is regulated through the following affine control system:

$$
\dot{X}(t)=F^{0}(X(t))+\sum_{(i, j) \in \operatorname{Diff}(t)} F^{(i, j)}(X(t)) \cdot u_{(i, j)}(t), \quad X(t) \in M(t) .
$$

where the state space $M(t)=\mathbb{R}_{+}^{\operatorname{dim}(\operatorname{Diff}(t))}$ varies with time, the vector fields $F_{0}, F^{(i, j)}$ are given respectively by equations $(3)$ and $(5,6)$, and such that $u($.$) is an admissible control. What is unusual in the considered problem$ with respect to traditional control problems is that the initial and final conditions are given in terms of $\operatorname{Cell}(0)$ and $\operatorname{Cell}(T)$ rather than in terms of $X(0), X(T)$.

More precisely, we have:

Problem $\alpha$ : Given A, Cell $(0)$ and $\operatorname{Cell}(T)$, determine $X(0)$ and an admissible control $u($.$) such that Cell(0) transforms into Cell(T) under the$ evolution of system (2) and the prescribed rules for mitosis.

Notice that the admissible control is determined by the fractone set Fract $(t)$ at almost every time $t \in[0, T]$. 

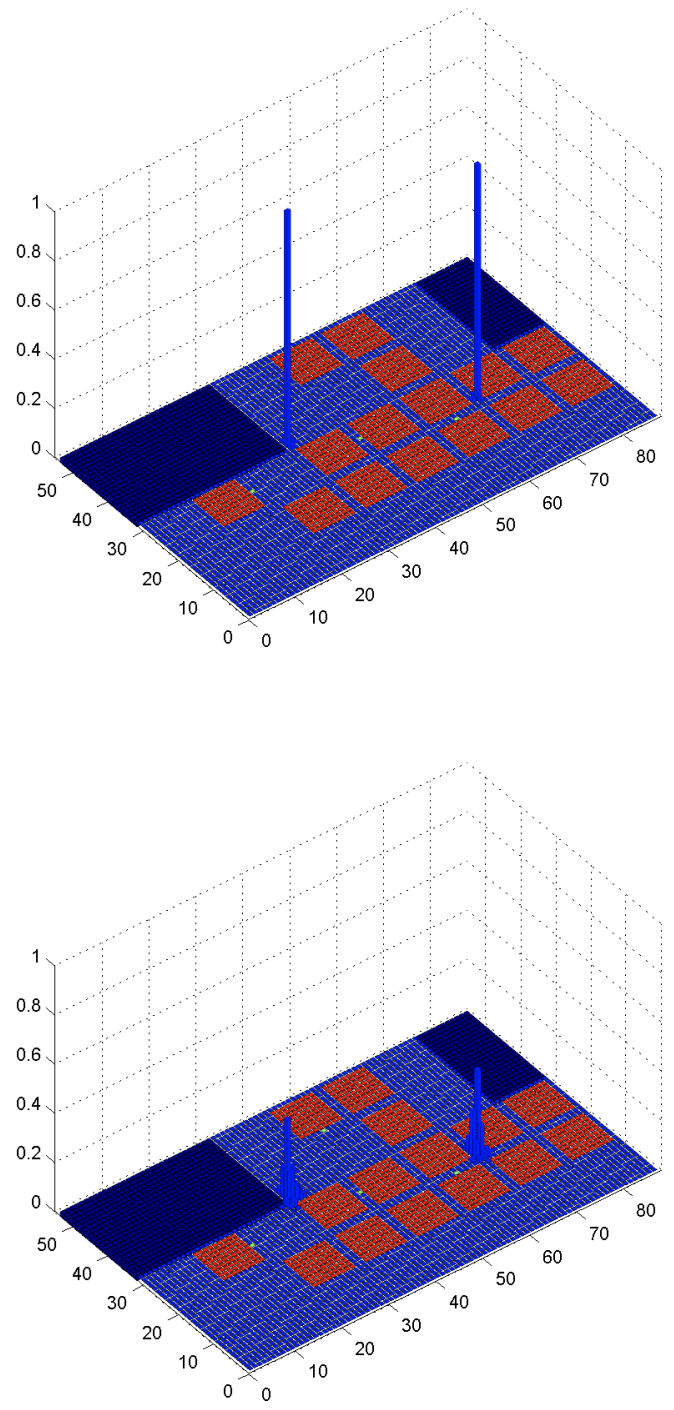

Figure 6: On the top figure, we can see two single initial sources of GF (located at exactly two units of our discretization). The bottom figure shows diffusion of the two sources in the neighboring units in $\operatorname{Diff}(t)$ while avoiding the units in $\operatorname{Cell}(t)$. 


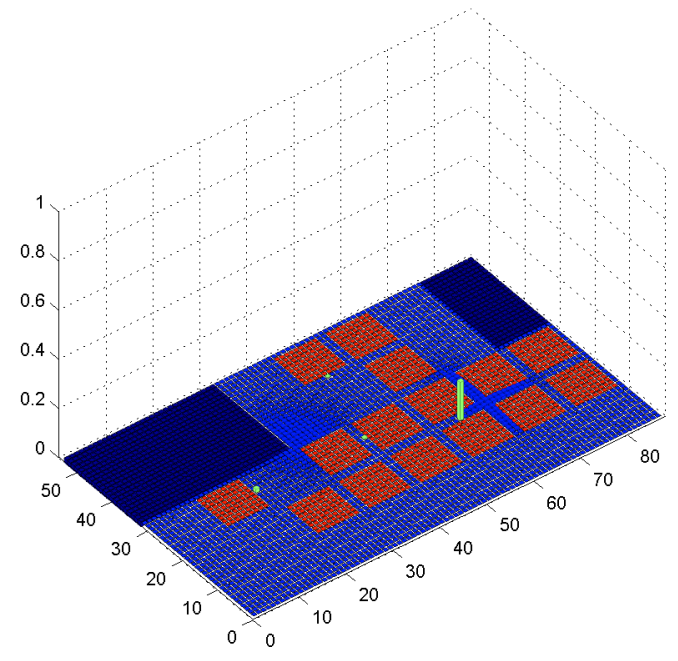

Figure 7: This figure displays the fact that as the diffusion process advances the acrtive fractones capture the GF, this can be seen with the quantify of growth fractones growing above the active fractones represented in green. 


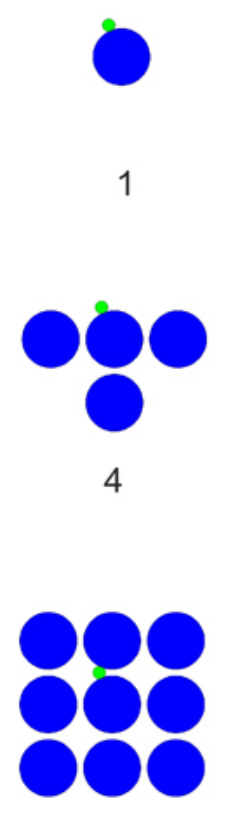

7

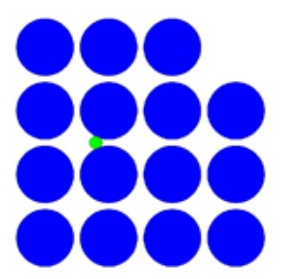

10

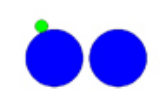

2

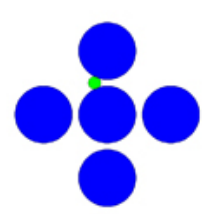

5

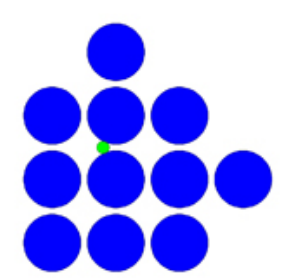

8

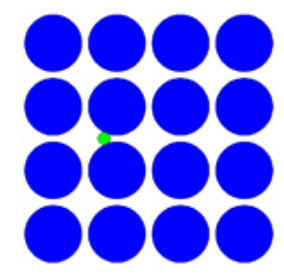

11

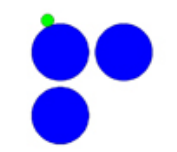

3

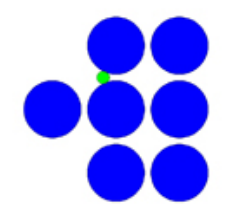

6

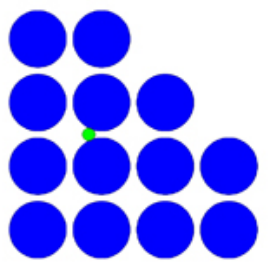

9

Figure 8: Starting from a unit cell and a single fractone that spans two units, the sequence of images illustrate the deformation algorithm as duplication of the cell associated to the fractone occur. If we choose the origin such that the center of the initial cell is at $(0,0)$. we have that the initial cell space (Figure 8,1) is $\{(i, j) \in \mathbb{Z} \times \mathbb{Z} \mid-4 \leq i \leq 4,-4 \leq j \leq 4\}$, and the final cellspace (Figure $\mathbf{8 , 1 1})$ is $\{(i, j) \in \mathbb{Z} \times \mathbb{Z} \mid-26 \leq i \leq 26,-26 \leq j \leq 26\} \backslash\{(i, j) \mid i \in\{-6,-5,5,6,16,17\}, j \in$ $\{-6,-5,5,6,16,17\}\}$. 


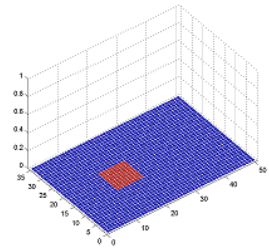

(a)

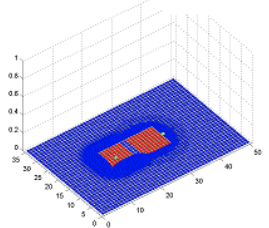

(d)

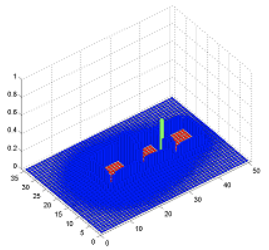

(g)

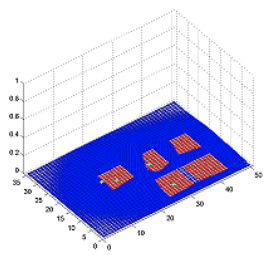

(j)

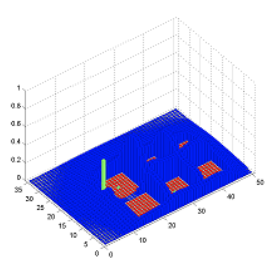

(m)

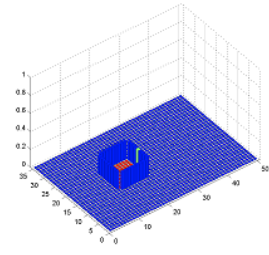

(b)

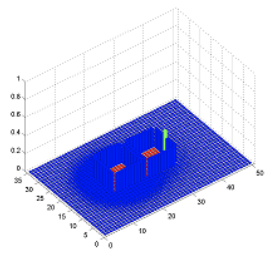

(e)

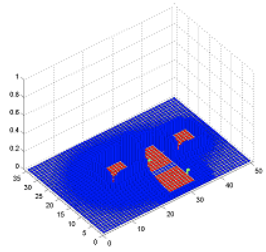

(h)

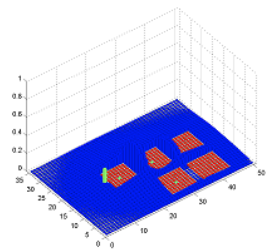

(k)

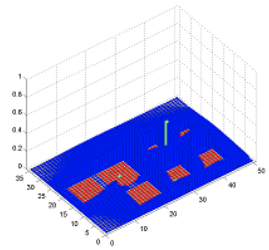

(n)

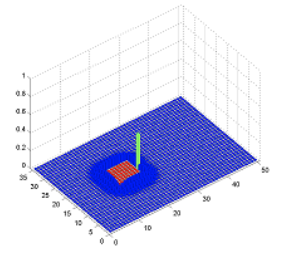

(c)

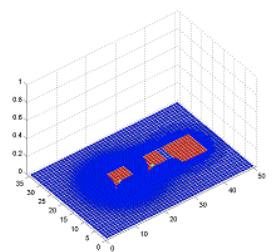

(f)

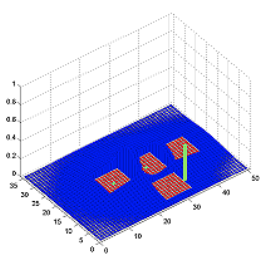

(i)

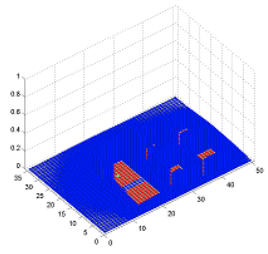

(I)

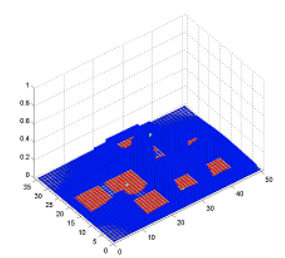

(o)

Figure 9: Cell space growth, with growth factor production, diffusion, and capture by fractones. Fractones are activated and deactivated between mitosis events.

São Paulo J.Math.Sci. 5, 2 (2011), 281-315 


\section{Theoretical Results}

In this section, we analyze some theoretical questions related to the mathematical problem $\alpha$ stated in Section 2.5.2. The key element in our model is the role played by the fractones as controllers. Under our hypothesis, they regulate cell's proliferation and differentiation. Growth factor intervenes in cell proliferation, but the fractones are the mechanism guiding and regulating GF. In our model, the production and diffusion of GF determines the time (or equivalently the order) at which the morphogenic events take place but it is the fractones that control the process. For instance, production of GF can always be altered such that a given active fractone will reach the GF threshold at a precise time. Moreover, the results presented in this section are based on having a unique active fractone at a time. Therefore, the diffusion and production of GF does not play any role in generating the morphogenic events (it only provides temporal information). For this reason, in this section, we consider the simplified problem where we neglect the GF diffusion and focus on how spatial distribution of fractones regulate cell proliferation. Once again, this is not a restrictive simplification and our results can be simulated using the complete model.

The results presented in this section are based on the assumptions made in our examples, in particular we consider the algorithm for deformation of the mass of cells described in Example 3.

The first two results deal with existence and uniqueness of solutions for our mathematical problem.

Lemma 1. There exist choices of $A, \operatorname{Cell}(0)$ and $\operatorname{Cell}(T)$ such that we cannot find an initial concentration of growth factor $X(0)$ as well as an admissible control to solve Problem $\alpha$.

Proof. This result is a consequence of our choice for the rules governing the deformation of the cell space once mitosis happens. Figure 10 provides an example.

Lemma 2. Assume $A$, Cell(0) and $\operatorname{Cell}(T)$ are such that there exists $X(0)$ and an admissible control such that there is a solution to Problem $\alpha$. The solution might not be unique.

Proof. It is straightforward to produce an example; see Figure 11 for an illustration.

Lemmas 1 and 2 suggest two lines of work. First, since we might not be able to reach a prescribed final cell configuration a natural questions is then: 


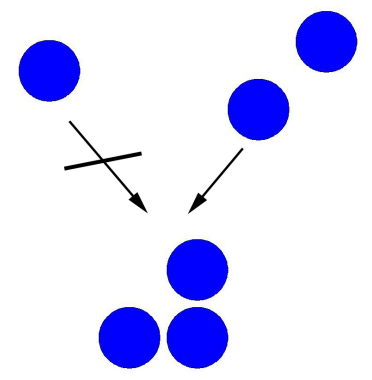

Figure 10: Example of a final configuration that can be attained using our algorithm from the initial configuration on the right with two cells but not from the single cell configuration on the left.

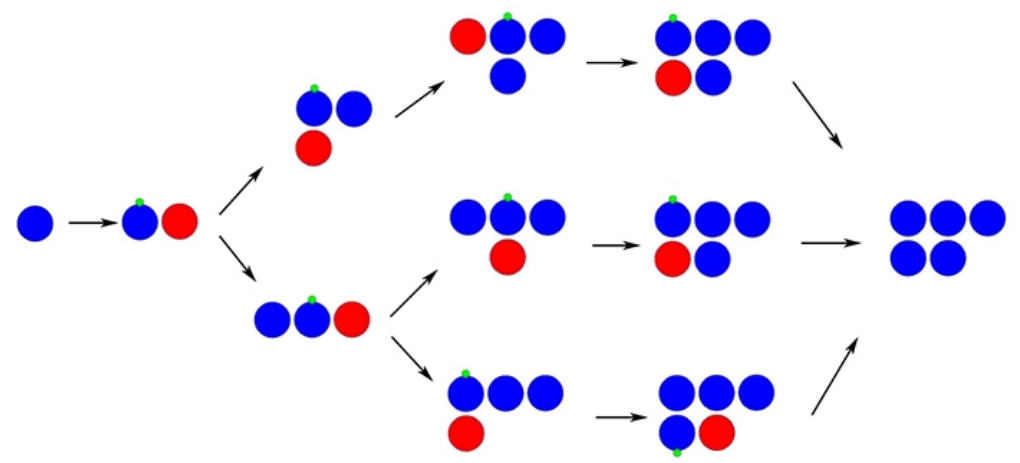

Figure 11: Illustration of several sequences of morphogenic events starting from a unique cell and reaching the same final configuration.

How close can we reach a prescribed final configuration? Second, the nonuniqueness of the solution when it exists raises the question of optimality. In this paper, we will focus on the first aspect, i.e. on the reachable set of configurations. Optimization of the solutions will be addressed in a forthcoming paper. Before we can state some results we need to introduce some definitions. In order to measure how close two configurations of cells are from each other we use the Hausdorff distance. In the sequel, all spaces are identified to their corresponding discretization and a given ambient space $A$ has been prescribed in which all the considered mass of cells live. 
Definition 8. Given a cell space Cell $\subset$ A, an element of Cell corresponds to a biological cell. In our discretization, it is identified to a single unit representing the cell. In Example 1, an element (i.e. a cell) a $\in$ Cell is a $9 \times 9$ square identified to its middle unit $\left(i_{a}, j_{a}\right)$ and we write $C_{a}=$ $\left(i_{a}, j_{a}\right) \in$ Cell. Under our assumption that our cells are horizontally and vertically aligned, we have given two cells $C_{a}, C_{b} \in$ Cell that $d_{E}\left(C_{a}, C_{b}\right) \in$ $\left\{11 \sqrt{n^{2}+m^{2}} \mid n, m \in \mathbb{Z}\right\}$. For this section, we introduce a new distance $d_{C}$ :

$$
d_{C}\left(C_{a}, C_{b}\right)=d_{E}\left(C_{a}, C_{b}\right) / 11
$$

so that two adjacent cells are at a distance 1 and not 11 (the 11 being solely dependent on the discretization and therefore misleading). Two diagonal cells are therefore at a distance $\sqrt{2}$.

Definition 9. Let Cell $_{A}$, Cell $_{B} \subset A$ be two cell spaces.

(1) We define the directed Hausdorff distance, $d$, by:

$$
d\left(\text { Cell }_{A}, \text { Cell }_{B}\right)=\max _{C_{a} \in \operatorname{Cell}_{A}} \min _{C_{b} \in \operatorname{Cell}_{B}} d_{C}\left(C_{a}, C_{b}\right)
$$

Thus $d\left(\mathrm{Cell}_{A}, \mathrm{Cell}_{B}\right)$ gives the minimum distance from the cell $C_{a} \in \mathrm{Cell}_{A}$ to any cell in $\mathrm{Cell}_{B}$, where $C_{a}$ is the cell in $\mathrm{Cell}_{A}$ furthest from any cell in $\mathrm{Cell}_{B}$.

(2) The Hausdorff distance, $D_{H}$, is given by:

$$
D_{H}\left(\operatorname{Cell}_{A}, \operatorname{Cell}_{B}\right)=\max \left(d\left(\operatorname{Cell}_{A}, \operatorname{Cell}_{B}\right), d\left(\operatorname{Cell}_{B}, \text { Cell }_{A}\right)\right)
$$

Example 4. Consider the cell spaces in Fig. 12. With the origin placed in the bottom left of the ambient space, we let

$$
\begin{aligned}
& \text { Cell }_{A}= \\
& \{(i, j) \mid i \in\{12,13, \ldots, 20\}, j \in\{1,2, \ldots, 9,12,13, \ldots, 20,23,24, \ldots, 31\}\} \bigcup \\
& \{(i, j) \mid i \in\{23,24, \ldots 31\}, j \in\{12,13, \ldots, 20\}\}
\end{aligned}
$$

and

$$
\begin{aligned}
\text { Cell }_{B}= & \{(i, j) \mid i, j \in\{1,2, \ldots, 9\}\} \bigcup\{(i, j) \mid i, j \in\{23,24, \ldots, 31\}\} \bigcup \\
& \{(i, j) \mid i \in\{34,35, \ldots, 42,45,46, \ldots, 53\}, j \in\{12,13, \ldots, 20\}\} .
\end{aligned}
$$

An easy calculation shows that $d\left(\mathrm{Cell}_{A}, \mathrm{Cell}_{B}\right)=\sqrt{2}, d\left(\mathrm{Cell}_{B}, \mathrm{Cell}_{A}\right)=2$, and $D_{H}\left(\mathrm{Cell}_{A}, \mathrm{Cell}_{B}\right)=2$.

The next definition introduces the notion of a walk between two biological cells. 


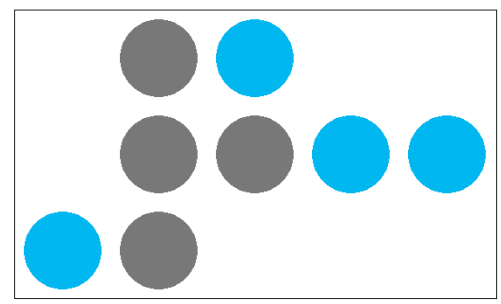

(a)

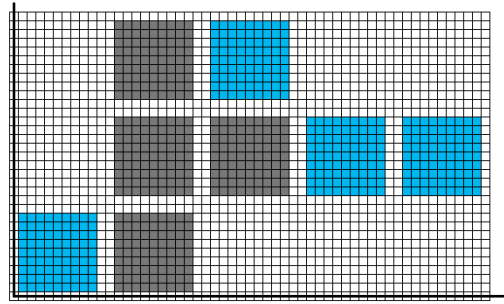

(b)

Figure 12: (a) The cells in $C e l l_{A}$ and $C e l l_{B}$ represented by circles. (b) The discretization of $C e l l_{A}$ and $C e l l_{B}$ with axes included.

Definition 10. Let $C_{a}, C_{b}$ be two cells. A walk from $C_{a}$ to $C_{b}$ is a sequence of cells, $\left\{C_{a_{i}}\right\}_{i=0}^{n}$, such that $C_{a}=C_{a_{0}}, C_{b}=C_{a_{n}}$ and $d_{C}\left(C_{a_{j}}, C_{a_{j+1}}\right)=1$ for $j=0,1,2, \ldots, n-1$. A walk $\left\{C_{a_{i}}\right\}_{i=0}^{n}$ is minimal if

$$
\begin{aligned}
\max _{j=0,1,2, \ldots, n} \min \left(d_{C}\left(C_{a_{0}}, C_{a_{j}}\right), d_{C}\left(C_{a_{j}}, C_{a_{n}}\right)\right) \\
\leq \max _{j=0,1,2, \ldots, m} \min \left(d_{C}\left(C_{b_{0}}, C_{b_{j}}\right), d_{C}\left(C_{b_{j}}, C_{b_{m}}\right)\right)
\end{aligned}
$$

for any other walk $\left\{C_{b_{i}}\right\}_{i=0}^{m}$ with $C_{b_{0}}=C_{a}, C_{b_{m}}=C_{b}$.

Clearly, a walk must "cross" the line equidistant (using the Euclidian metric) to the two end-point cells $C_{a}$ and $C_{b}$. We will call any cell through which this line passes a "middle cell". Notice that for some scenarios, there might not be any middle cell (see Figure 13a). This can be the case only if the first or second indices of the cells $C_{a}$ and $C_{b}$ coincide. Assume that $C_{a}$ and $C_{b}$ are not aligned, then middle cells exists and it is clear that a minimal walk must contains a middle cell such that the sum of its distance to $C_{a}$ and its distance to $C_{b}$ is less or equal to this sum for any other middle cell. It is also true that this distance represents the value $\max _{j=0,1,2, \ldots, n} \min \left(d_{C}\left(C_{a_{0}}, C_{a_{j}}\right), d_{C}\left(C_{a_{j}}, C_{a_{n}}\right)\right.$ ) (see Figure 13).

Proposition 1. Let $C_{1}$ and $C_{2}$ be two cells in the ambient space. Then, there exists a minimal walk from $C_{1}$ to $C_{2}$. The minimal walk might not be unique.

Proof. The proof is based on our previous remarks. First let us introduce a specific construction for a walk between $C_{1}=\left(i_{1}, j_{1}\right)$ and $C_{2}=$ $\left(i_{2}, j_{2}\right)$ and then we will prove that it is minimal. We introduce $d_{i}=$ $\left\{\begin{array}{lll}\left\lfloor\frac{i_{2}-i_{1}}{2}\right\rfloor & \text { if } & i_{2}-i_{1} \geq 0 \\ \left\lceil\frac{i_{2}-i_{1}}{2}\right\rceil & \text { if } & i_{2}-i_{1}<0\end{array}\right.$ and 


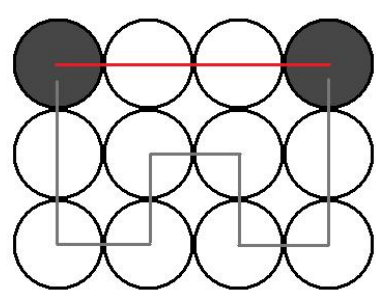

(a)

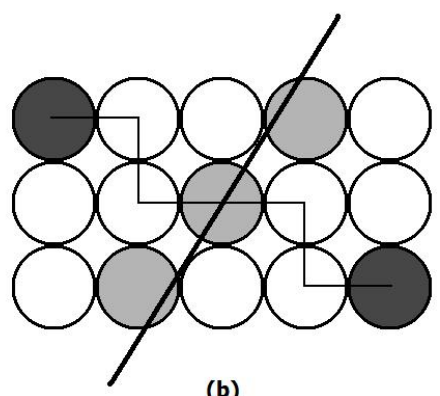

(b)

Figure 13: (a) Two example walks, one of which is a minimal walk. (b) A minimal walk, with middle cells highlighted. Note that any minimal walk must pass through the middle cell in the center, and will not pass through any other middle cell.

$d_{j}=\left\{\begin{array}{lll}\left\lfloor\frac{j_{2}-j_{1}}{2}\right\rfloor & \text { if } \quad j_{2}-j_{1} \geq 0 \\ \left\lceil\frac{j_{2}-j_{1}}{2}\right\rceil & \text { if } \quad j_{2}-j_{1}<0\end{array}\right.$ as the horizontal and vertical integer part distances. If $d_{i} \leq 2$ and $d_{j} \leq 2$, the minimal walk is straightforward to construct. Otherwise, we construct a minimal walk inductively by finding a minimal walk between $\left(i_{1}, j_{1}\right)$ and $\left(i_{1}+d_{i}, j_{1}+d_{j}\right)$ and a minimal walk between $\left(i_{2}-d_{i}, j_{2}-d_{j}\right)$ and $\left(i_{2}, j_{2}\right)$ (if the horizontal and vertical integer part distances between these two indices are greater than 2 we keep subdiving). We concatenate these minimal walks to achieve a walk between $\left(i_{1}, j_{1}\right)$ and $\left(i_{2}, j_{2}\right)$. This final walk is minimal. Indeed, its middle point is the furthest unit in the walk from either $a_{1}$ or $a_{2}$ but by construction it is also the closest cell from $a_{1}$ and $a_{2}$ among the set of all middle cells (and this is also true for each of the subdivision).

Notice that it is not true that the concatenation of two minimal walks is always also a minimal walk.

Definition 11. A cell space Cell is called 1-connected if for any two cells, $C_{a}, C_{b} \in$ Cell, there exists a walk $\left\{C_{a_{i}}\right\}$ with $C_{a_{i}} \in$ Cell for all $i$ and $C_{a_{0}}=C_{a}, C_{a_{n}}=C_{b}$. More generally, Cell is said to be $r$-connected if there exist a sequence $\left\{C_{a_{i}}\right\}_{i=0}^{n}$ with $C_{a_{i}} \in$ Cell for all $i, C_{a_{0}}=C_{a}, C_{a_{n}}=C_{b}$ and such that $d_{C}\left(C_{a_{j}}, C_{a_{j+1}}\right) \leq r$ for $j=0,1,2, \ldots, n-1$. We call Cell minimally $r$-connected if Cell is $r$-connected but not $m$-connected for any $m<r$.

Definition 12. Let $\mathrm{Cell}_{A}$ and $\mathrm{Cell}_{B}$ be two cell spaces. A path (when it exists) between $\mathrm{Cell}_{A}$ to $\mathrm{Cell}_{B}$ is a sequence of morphogenic events such 


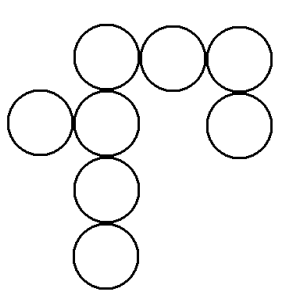

(a)

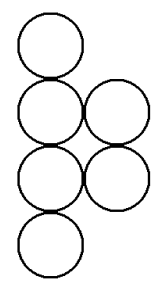

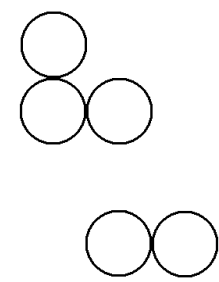

(b)

Figure 14: (a): 1-connected cell space. (b): Minimally 3-connected cell space

that $\mathrm{Cell}_{A}$ deforms into $\mathrm{Cell}_{B}$. More precisely, the existence of a path is equivalent to the existence of an initial value $X(0)$ and an admissble control $u($.$) defined on [0, T], \operatorname{Cell}_{A}=\operatorname{Cell}(0)$ such that there is a solution to problem $\alpha$ with $\operatorname{Cell}_{B}=\operatorname{Cell}(T)$. Given the fact that in this section we neglect the role of the growth factor, it boils down to the existence of a dynamic spatial distribution of fractones that generates the desired growth.

Proposition 2. Given an initial cell space Cell(0) and a 1-connected cell space $\mathrm{Cell}_{F}$ such that $\mathrm{Cell}(0) \subset \mathrm{Cell}_{F}$, then there exists a path from $\operatorname{Cell}(0)$ to $\operatorname{Cell}(T)$ for some $T$ such that $D_{H}\left(\operatorname{Cell}(T), \operatorname{Cell}_{F}\right) \leq 1$.

Proof. Since Cell(0) is assumed to be a strict subset of $\operatorname{Cell}(T)$, let $C_{a_{0}} \in$ $\operatorname{Cell}(0)$ such that there exists $C_{a_{1}} \in \operatorname{Cell}(T)$ with $d_{C}\left(C_{a_{0}}, C_{a_{1}}\right)=1$ and $C_{a_{1}} \notin \operatorname{Cell}(0)$ (ie. $C_{a_{1}}$ must be directly above, below, to the right, or to the left of $C_{a_{0}}$ ). This is possible since $\operatorname{Cell}(T)$ is 1-connected. We activate a fractone associated to the cell $C_{a_{0}}$ to induce mitosis. If $C_{a_{1}}$ is to the right of $C_{a_{0}}$, then when growth is triggered, the mass of cell deforms and $C_{a_{1}}$ is brought into the new cell space. If $C_{a_{1}}$ is below, to the left, or above $C_{a_{0}}$, then growth must occur at least two, three, or four times, respectively, before the mass of cell deforms in the $C_{a_{1}}$ direction. The extra cells created in this growth will be distance 1 from $C_{a_{0}}$, and therefore either in $\mathrm{Cell}_{F}$ or a distance 1 from Cell $_{F}$. Inductively, we inactivate the fractone and repeat the process. When no cell $C_{a_{0}}$ satisfying our assumptions exists, then the newly obtained cell space at that time, $T$, must be within Hausdorff distance 1 since no extra cell created was more than distance 1 from any element of $\mathrm{Cell}_{F}$.

The next natural question is to determine how to modify the deformation algorithm in order to replace the existence of a path from Cell(0) to $\operatorname{Cell}(T)$ for some $T$ such that $D_{H}\left(\operatorname{Cell}(T), \operatorname{Cell}_{F}\right) \leq 1$ in Proposition 2 by 


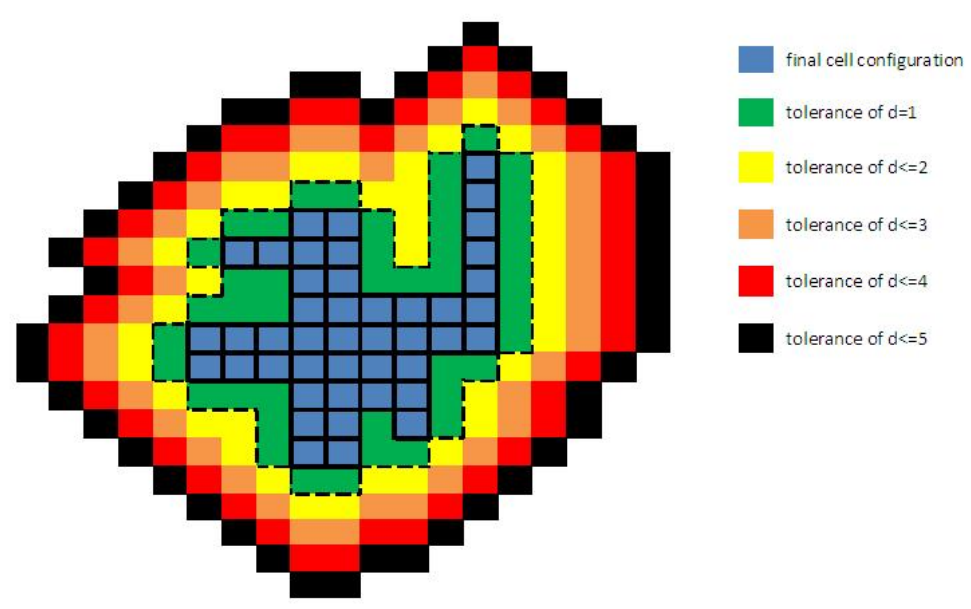

Figure 15: Proposition 2 states that from any initial cell space included in the final cell space represented in blue can be deformed into a cell space strictly included in the green space at Hausdorff distance 1 of the desired final cell space.

the existence of a path such that $\operatorname{Cell}(T)=\operatorname{Cell}_{F}$, i.e. we can reach exactly the final prescribed cell space. Proposition 3 states that we can improve the accuracy of the growth if we allow rotation of the entire configuration space between growth events. This is not suprising since it essentially allows us to choose the direction of cell growth.

Proposition 3. Given an initial cell space Cell(0) and a 1-connected cell space $\mathrm{Cell}_{F}$ such that $\operatorname{Cell}(0) \subset \mathrm{Cell}_{F}$, then, with rotations of the configuration space allowed between morphogenic events, there exists a path from $\operatorname{Cell}(0)$ to $\operatorname{Cell}(T)$ for some $T$ such that $D_{H}\left(\operatorname{Cell}(T), \operatorname{Cell}_{F}\right)=0$.

Proof. As in Proposition 2, let $C_{a_{0}} \in \operatorname{Cell}(0)$ such that there exists $C_{a_{1}} \in$ $\operatorname{Cell}(T)$ with $d_{C}\left(C_{a_{0}}, C_{a_{1}}\right)=1$ and $C_{a_{1}} \notin \operatorname{Cell}(0)$. Rotate $\operatorname{Cell}(0)$ and Cell $F$ together until $C_{a_{1}}$ (or its rotational image) lies to the right of $C_{a_{0}}$ (or its rotational image). We then proceed exactly as in Proposition 2 by activating a fractone associated to cell $C_{a_{0}}$. The cell space is then deformed exactly in the $C_{a_{1}}$ direction. Since Cell $_{F}$ is connected, this process can be repeated, and the cell space rotated back to its original orientation, until $D_{H}\left(\operatorname{Cell}(T), \operatorname{Cell}_{F}\right)$. 
One should note this path from $\operatorname{Cell}(0)$ to $\operatorname{Cell}(T)$ is neither unique nor time-optimal. In fact, it is quite slow as at most 1 fractone is active at any given time. Notice also that from the biological point of view, a deformation algorithm that allows translation and rotation of the cell space is not realistic for several reasons. It is interesting purely from a mathematical point of view.

The next result concerns a cell growth from an initial cell space to reach as close as possible a final cell space that is minimally $r$-connected, $r>1$. Let us first state a useful lemma.

LEMmA 3. Let $C_{1}=\left(i_{1}, j_{1}\right)$ and $C_{2}=\left(i_{2}, j_{2}\right)$ be two cells in a cell space such that $d_{C}\left(C_{1}, C_{2}\right)=r, r$ not even. Then for the middle point $C_{a_{m}}=\left(i_{m}, j_{m}\right)$ of a minimal walk between $C_{1}$ and $C_{2}, \min \left(d_{C}\left(C_{1}, C_{a_{m}}\right), d_{C}\left(C_{a_{m}}, C_{2}\right)\right) \leq$ $\frac{r}{\sqrt{2}}$.

Proof. Let $d_{i}=\frac{1}{11}\left|i_{2}-i_{1}\right|$ and $d_{j}=\frac{1}{11}\left|j_{2}-j_{1}\right|$. Then $r=\sqrt{d_{i}^{2}+d_{j}^{2}}$. We first note that the case where either $d_{i}$ or $d_{j}$ equal 0 is trivial. The minimal walk is a straight row or column, and the distance to a middle cell is equal to $\frac{r}{2}$ (if $r$ is even there is no middle cell). If $d_{i}=1$ and $d_{j}=1$, then it is simple to verify that $r=\sqrt{2}$ and the distance to the middle points is 1 . Otherwise, in general, there are three cases: (i) $d_{i}, d_{j}$ both even; (ii) one of $d_{i}, d_{j}$ odd; (iii) or both $d_{i}, d_{j}$ odd. Case (i): If $d_{i}, d_{j}$ both even, then the middle point is a distance $\sqrt{\left(\frac{d_{i}}{2}\right)^{2}+\left(\frac{d_{j}}{2}\right)^{2}}=r / 2<\frac{r}{\sqrt{2}}$ from either $C_{1}$ or $C_{2}$. Case (ii): Without loss of generality, suppose $d_{i}$ is odd and $d_{j}$ is even. Then the furthest distance from the middle point of a minimal walk to either $C_{1}$ or $C_{2}$ will be $\sqrt{\left(\frac{d_{i}+1}{2}\right)^{2}+\left(\frac{d_{j}}{2}\right)^{2}}=\frac{1}{2} \sqrt{d_{i}^{2}+d_{j}^{2}+2 d_{i}+1} \leq \frac{1}{\sqrt{2}} \sqrt{d_{1}^{2}+d_{j}^{2}}$ if $2 d_{i}+1 \leq d_{i}^{2}+d_{j}^{2}$. Since $d_{i} \geq 2$ and $d_{j} \geq 1$ (else we would be in one of the previously proven cases), the inequality holds. Case (iii): If both $d_{i}, d_{j}$ are odd (note in this case $d_{i} \geq 3$ and $d_{j} \geq 3$ ), then the furthest distance from the middle point of a minimal walk to either $C_{1}$ or $C_{2}$ is $\sqrt{\left(\frac{d_{i}+1}{2}\right)^{2}+\left(\frac{d_{j}-1}{2}\right)^{2}}$. It follows that $\sqrt{\left(\frac{d_{i}+1}{2}\right)^{2}+\left(\frac{d_{j}-1}{2}\right)^{2}} \leq \frac{1}{\sqrt{2}} \sqrt{d_{1}^{2}+d_{j}^{2}}$ if $2 d_{i}-2 d_{j}+2<d_{i}^{2}+d_{j}^{2}$. Since $d_{i} \geq 3$ and $d_{j} \geq 3$, the inequality holds (the same argument is true if we invert $i$ and $j$ in the equations depending on the minimal walk that has been chosen).

Proposition 4. Given an initial cell space Cell(0) and a minimally $r$ connected cell space, $r>1$, Cell $_{F}=\bigcup_{i=1}^{n} \operatorname{Cell}_{F_{i}}$, where each Cell $_{F_{i}}$ is 1-connected. Assume $\mathrm{Cell}(0) \subset \mathrm{Cell}_{F_{k}}$ for some $k$. Then there exists a path from $\operatorname{Cell}(0)$ to $\operatorname{Cell}(T)$ for some $T$ such that $D_{H}\left(\operatorname{Cell}(T), \operatorname{Cell}_{F}\right) \leq \frac{r}{\sqrt{2}}$. 
Proof. First, we construct from Cell $_{F}$ a new cell space by bridging the gaps between all its components Cell $F_{i}$. The algorithm goes as follows. Find the cell in $\operatorname{Cell}_{F_{1}}$ and the cell in $\bigcup_{i \neq 1}$ Cell $_{F_{i}}$ that have minimal distance $d_{C}$ to each other. Find a minimal walk between these two cells, call it $\left\{C_{a_{1 i}}\right\}$. Without loss of generality, suppose we connect $\mathrm{Cell}_{F_{1}}$ and $\mathrm{Cell}_{F_{2}}$. Then find a cell in Cell $_{F_{1}} \cup$ Cell $_{F_{2}}$ and a cell in $\bigcup_{i>2}$ Cell $_{F_{i}}$ that minimize the distance $d_{C}$ between all cells in those two cell spaces. Again, find a minimal walk between these two cells, call it $\left\{C_{a_{2 i}}\right\}$. Proceed iteratively until all Cell $F_{i}$ have walks, $\left\{C_{a_{j i}}\right\}$, between them. Then let $\mathrm{Cell}_{G}$ be the union of $\operatorname{Cell}_{F}$ and $\bigcup_{j=1}^{n-1}\left\{C_{a_{j i}}\right\}$. Then Cell $G$ is 1-connected, so by previous Proposition, there exists a path from $\operatorname{Cell}(0)$ to $\operatorname{Cell}(T)$ for some $T$ such that $D_{H}\left(\operatorname{Cell}(T), \operatorname{Cell}_{G}\right) \leq 1$. The difference between $\operatorname{Cell}_{F}$ and $\operatorname{Cell}_{G}$ are the minimal walks, so the Hausdorff distance from $\operatorname{Cell}(T)$ to $\operatorname{Cell}_{F}$ will be at most the maximum distance from a walk endpoint to a point $\sqrt{2}$ away from the closest middle cell. Since $\mathrm{Cell}_{F}$ is minimally $r$-connected, we need only to consider the walks between cells at a distance $r$ from each other. Let $C_{1}=\left(i_{1}, j_{1}\right)$ and $C_{2}=\left(i_{2}, j_{2}\right)$ be two such cells. Let $d_{i}=\frac{1}{11}\left|i_{2}-i_{1}\right|$ and $d_{j}=\frac{1}{11}\left|j_{2}-j_{1}\right|$. Then $r=\sqrt{d_{i}^{2}+d_{j}^{2}}$. Suppose first that $d_{i}$ is 0 . We note $d_{j}$ cannot equal one since we assume $\operatorname{Cell}_{F}$ is not 1-connected. If $d_{j} \geq 3$ is odd, then the minimal walk between $C_{1}$ and $C_{2}$ is a column of cells, and the minimum of the maximum distances to a cell one away (left or right) from this walk is given by $\sqrt{\left(\frac{d_{j}-1}{2}\right)^{2}+1}$. This will be less than or equal to $\frac{r}{\sqrt{2}}$ if $5 \leq d_{j}^{2}+2 d_{j}$, which is true for $d_{j} \geq 3$. Similarly, if $d_{j} \geq 2$ is even, then the distance to a cell one away from the middle cell is: $\sqrt{\left(\frac{d_{j}}{2}\right)^{2}+1}$. Note that $\sqrt{\left(\frac{d_{j}}{2}\right)^{2}+1} \leq \frac{r}{\sqrt{2}}$ if $4 \leq$ $d_{j}^{2}$, which is always true for $d_{j} \geq 2$. Remark that the above argument applies even if the values of $d_{i}$ and $d_{j}$ are swapped (covering the case where the minimal walk is a horizontal row of cells), and covers the worst-case scenario; clearly, growing to the right along a horizontal minimal walk will match the minimal walk exactly, giving a Hausdorff distance within $r / 2$, while growing to the left requires you to pass through a middle cell above or below the middle cell of the minimal walk. Suppose now that neither $d_{i}$ nor $d_{j}$ are 0 . If both $d_{i}$ and $d_{j}$ are even, then the minimal walk must pass through the middle cell $M_{1}=\left(i_{1} \pm \frac{d_{i}}{2}, j_{1} \pm \frac{d_{j}}{2}\right)$, where the \pm is determined by the relative position of $C_{2}$ to $C_{1}$. Then the maximum distance from an endcell of the walk (ie. $C_{1}, C_{2}$ ) to a cell within $\sqrt{2}$ of $m_{1}$ is $\sqrt{\left.\left(\frac{d_{i}}{2}+1\right)^{2}+\left(\frac{d_{j}}{2}-1\right)^{2}\right)}$ (or equivalently we can interchange $i$ and 
$j)$. Moreover, $\sqrt{\left.\left(\frac{d_{i}}{2}+1\right)^{2}+\left(\frac{d_{j}}{2}-1\right)^{2}\right)} \leq \frac{r}{\sqrt{2}}$ if $8 \leq\left(d_{i}^{2}-4 d_{i}\right)+\left(d_{j}^{2}+4 d_{j}\right)$, which is always true for $d_{i}, d_{j} \geq 2$. The case of $d_{i}=d_{j}=1$ is trivial. So suppose $d_{i}$ and $d_{j}$ are both odd and not both 1 . Then there are two middle cells the minimal walk could pass through. Without loss of generality, let it pass through $\left(i_{1}+\frac{d_{i}+1}{2}, j_{1}+\frac{d_{j}-1}{2}\right)$. Then the neighboring cells of interest are $N_{1}=\left(i_{1}+\frac{d_{i}-1}{2}, j_{1}+\frac{d_{j}+1}{2}\right)$ and $N_{2}=\left(i_{1}+\frac{d_{i}+3}{2}, j_{1}+\frac{d_{j}-3}{2}\right)$. The distance from $C_{1}$ to $N_{1}$ is $\sqrt{\left.\left(\frac{d_{i}+1}{2}\right)^{2}+\left(\frac{d_{j}-1}{2}\right)^{2}\right)}$. And this is less than $\frac{r}{\sqrt{2}}$ if $2 \leq\left(d_{i}^{2}-2 d_{i}\right)+\left(d_{j}^{2}+2 d_{j}\right.$, which is always true for $d_{i}, d_{j} \geq 1$. The distance from $C_{1}$ to $N_{2}$ is $\sqrt{\left.\left(\frac{d_{i}+3}{2}\right)^{2}+\left(\frac{d_{j}-3}{2}\right)^{2}\right)}$. And this is less than $\frac{r}{\sqrt{2}}$ if $18 \leq\left(d_{i}^{2}-6 d_{i}\right)+\left(d_{j}^{2}+6 d_{j}\right)$, which is always true for $d_{i}, d_{j} \geq 3$. It fails in the cases when $d_{i} \leq 7$ and $d_{j}=1$, however in these cases, it turns out that $N_{2}$ is closer to $C_{2}$ than to $C_{1}$ and it is easy to show that a walk can still be grown that satisfies the desired conclusion (see Figure 16). Finally suppose now, without loss of generality, that $d_{i}$ is odd and $d_{j}$ is even. Assume that one of the middle cells of the minimal walk is $M_{2}=\left(i_{1}+\frac{d_{i}-1}{2}, j_{1}+\frac{d_{j}}{2}\right)$ (other cases are similar). By symmetry, a second middle cell that the minimal walk must pass through is $M_{3}=\left(i_{1}+\frac{d_{i}+1}{2}, j_{1}+\frac{d_{j}}{2}\right)$. $M_{2}$ is closer to $C_{1}$ than to $C_{2}$, and similarly, $M_{3}$ is closer to $C_{2}$ than to $C_{1}$. Therefore, in calculating Hausdorff distance, we need only look at the distances between cells neighboring $M_{2}$ and $C_{1}$. By symmetry, these will be the same as the distances between points neighboring $M_{3}$ and $C_{2}$. The neighboring cells to $M_{2}$ are $N_{3}=\left(i_{1}+\frac{d_{i}-3}{2}, j_{1}+\frac{d_{j}+2}{2}\right)$ and $N_{4}=\left(i_{1}+\frac{d_{i}+1}{2}, j_{1}+\frac{d_{j}-2}{2}\right)$. The distance to $N_{3}$ is $\sqrt{\left.\left(\frac{d_{i}-3}{2}\right)^{2}+\left(\frac{d_{j}+2}{2}\right)^{2}\right)}$. This is less than or equal to $\frac{r}{\sqrt{2}}$ if $13 \leq\left(d_{i}^{2}+6 d_{i}\right)+\left(d_{j}^{2}-4 d_{j}\right)$, which holds except in the cases where $d_{i}=1$ and $d_{j} \leq 4$. However, as in the previous case, it is easy to show that a walk can still be grown that satisfies the desired conclusion. The distance to $N_{4}$ is $\sqrt{\left.\left(\frac{d_{i}+1}{2}\right)^{2}+\left(\frac{d_{j}-2}{2}\right)^{2}\right)}$. This is less than or equal to $\frac{r}{\sqrt{2}}$ if $5 \leq\left(d_{i}^{2}-2 d_{i}\right)+\left(d_{j}^{2}+4 d_{j}\right)$, which holds for $d_{i} \geq 1$ and $d_{j} \geq 2$.

Proposition 5. Given an initial cell space Cell(0) and a minimally $r$ connected cell space $\mathrm{Cell}_{F}=\bigcup_{i=1}^{n} \mathrm{Cell}_{F_{i}}$, where each $\mathrm{Cell}_{F_{i}}$ is 1-connected, such that $\mathrm{Cell}(0) \subset \mathrm{Cell}_{F_{k}}$ for some $k$, then, with rotations allowed between morphogenic events, there exists a path from $\operatorname{Cell}(0)$ to $\operatorname{Cell}(T)$ for some $T$ such that $D_{H}\left(\operatorname{Cell}(T), \operatorname{Cell}_{F}\right) \leq \frac{r}{\sqrt{2}}$. 

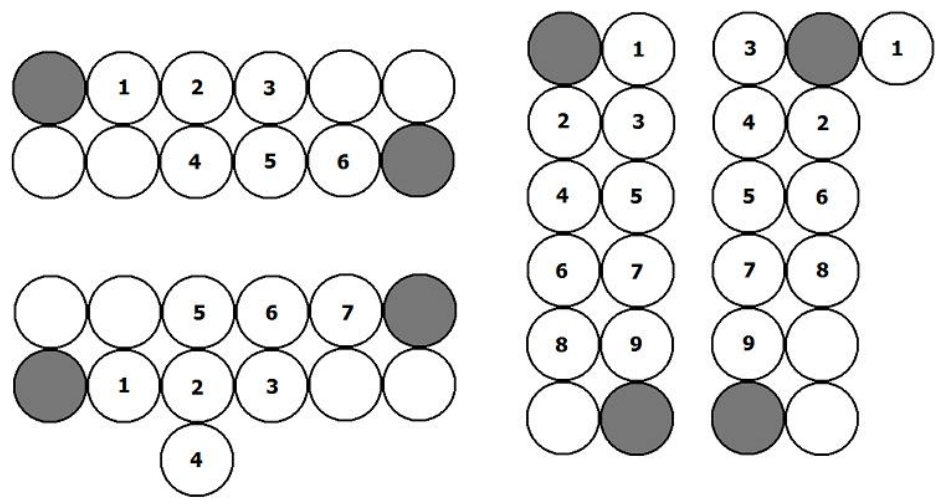

Figure 16: (a) The four possible configurations when $d_{i}=1, d_{j}=5$ or $d_{i}=5$, $d_{j}=1$. Cells are numbered by order of growth. $r=\sqrt{26}$. The Hausdorff distance in all four cases is $\sqrt{5}$.

Proof. As in the above proof, we construct a new cell space, Cell ${ }_{G}$, that bridges the gaps between each $\mathrm{Cell}_{F_{i}}$. From Proposition 3, there exists a path from $\operatorname{Cell}(0)$ to $\operatorname{Cell}(T)$ such that $D_{H}\left(\operatorname{Cell}(T), \operatorname{Cell}_{G}\right)=0 . \operatorname{Cell}_{F}$ differs from Cell $_{G}$ solely by the minimal walks. Thus $D_{H}\left(\operatorname{Cell}_{G}, \operatorname{Cell}_{F}\right)$ is the distance from the cell on a minimal walk which is furthest from any point in $\mathrm{Cell}_{F}$. This must be one of the middle cells of a minimal walk. Since Cell $_{F}$ is minimally $r$-connected, the longest minimal walk(s) must connect cells a distance $r$ apart. Therefore, by Lemma $3, D_{H}\left(\operatorname{Cell}_{G}, \operatorname{Cell}_{F}\right) \leq \frac{r}{\sqrt{2}}$.

In this section, we presented some answers to the one of the most basic question, which is the existence of a path between two given cell spaces. Clearly, much is still to be answered. For instance, when the existence of a path is established, we would like to determine a systematic way to compute an admissible control to solve problem $\alpha$. New techniques in control theory will need to be developed since our problem differs from the classical ones. This is much more complex than what is presented here. In a forthcoming work, we will also address the uniqueness question. A first intuition would be to use as many fractones as possible to speed up the sequence of morphogenic events, but examples can be constructed to illustrate that the use of more than one fractone is not more time efficient. A first step will be to actually determine a cost function to be minimized; 
indeed, time might not be the dominant factor in terms of the biological process.

\section{Application to Layout optimization}

As part of engineering design, layout optimization plays a critical role in the pursuit of optimal design. Layout optimization aims at finding the optimum distribution or layout of material within a bounded domain, called the design domain, that minimizes an objective or target function, while satisfying a set of constraints (see the recent monograph and reviews [2, 10, 23], and the references therein). The existing topology optimization methods rest on sound mathematical foundations (see, for example, $[4,2]$ ). And mathematically, the search space, where the optimization layout is defined, is a topological vector space of infinite dimension - usually a Sobolev space [4]. In practice, however, computational methods used to solve layout optimization can only store and compute finite amount of data. This limitation forces any numerical optimization methods to rely on approximations of the search space and of the admissible topology configurations. The popular SIMP method [2], for example, models the search space as discrete functions on the discretized design domain. In other words, each point represents the "pixel" of the desired blueprint of the optimal design. As a consequence, a good resolution of the design may require a large number of pixels, and these pixels model both void and solid regions.

Similarly to our computational methods, natural systems are also restricted to a finite encoding: the DNA. However, natural systems have devised a strikingly different solution to the finitude problem, where the DNA encodes a developmental program that when "compiled and executed" performs a sequence of tasks that develops the final structure in stages. The results are patterned, complex, and multi-scaled structures that perform multiple task functions and are generically resistant to damage.

The control model developed in this paper determines a cellular proliferation process that mimics the developmental stages of natural organisms. These laws can be evolved to respond to desired requirements, and thus be used to search for high-performing engineering layouts. One possibility is to use environment cues for crafting the control laws determining the placement of the fractones. For instance, in a problem to minimize the mass of material to sustain a load, the stress level in the cells may be used as a parameter controlling the creation of a fractone when the stress level on a cell surpass a fixed threshold. An exploratory result is presented in Figure 17, where it is shown the cellular division following the inclusion of a fractone on a cell when its level stress exceeded a preassigned limit. The result of the application of a simplified version of the procedure without the diffusion of the growth factor is shown in Figure 18 - note that in this form 


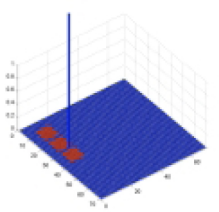

1

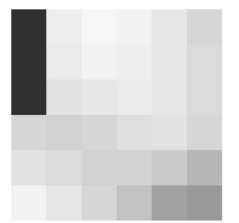

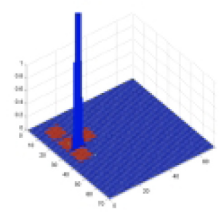

2

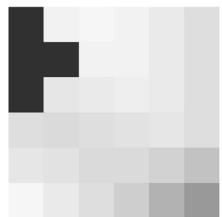

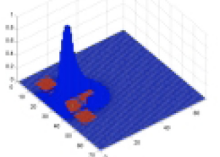

3

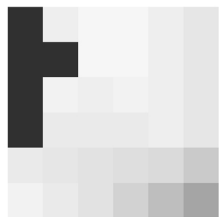

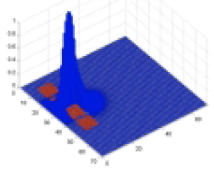

4

Figure 17: Stress-based law for fractone placement.

the method is similar to bi-directional evolutionary structural optimization methods (see e.g. [20]).

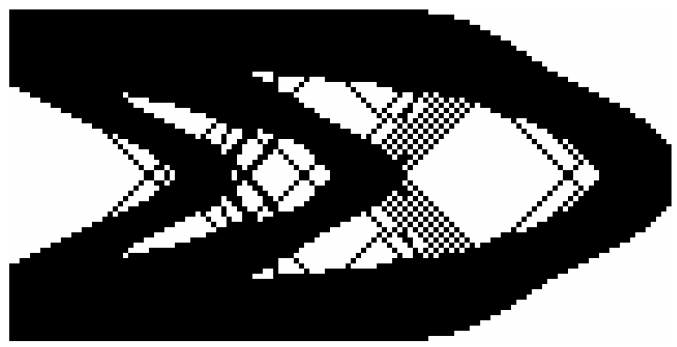

Figure 18: Preliminary result for layout optimization of a cantilever.

This approach will be developed in a forthcoming work. 


\section{References}

[1] Aviezer, D., Hecht, D., Safran, M., Elsinger, M. David, G., Yayon, A. Perlecan, basal lamina proteoglycan, promotes basic fibroblast growth factor-receptor bind-

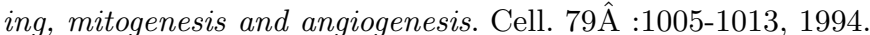

[2] M. P. Bendsøe, O. Sigmund, Topology Optimization: Theory, Methods and Applications, 2nd Edition, Springer, New York, 2003.

[3] Brickman, YG, Ford MD, Small DH, Bartlett PF, Nurcombe V. Heparan sulfates mediate the binding of basic fibroblast growth factor to a specific receptor on neural precursor cells. J. Biol. Chem. 270, 24941-24948, 1995.

[4] B. Bourdin, Filters in topology optimization, International Journal for Numerical Methods in Engineering 50 (2001) 2143-2158.

[5] Cattaneo E, McKay R.1990. Proliferation and differentiation of neuronal stem cells regulated by nerve growth factor. Nature 347:762-765.

[6] Autonomous Underwater vehicles Ocean Engineering, Special Issue on Autonomous Underwater Vehicles, Chyba, M.: Guest Editor, Vol 36/1, pp. 1-132, 2009.

[7] Chyba, M., Haberkorn, T., Smith, R.N., and Choi S.K. Autonomous Underwater Vehicles: Development and Implementation of time and Energy Efficient Trajectories. Ship Technology Research, 55/2, pp.36-48, 2008.

[8] Chyba, M., Mercier, F., Rader, J., Douet, V., Arikawa-Hirasawa, E., Kwon, Y.C., and Kodama, R.. Dynamic mathematical modeling of cell-fractone interactions. J. Math-for-Ind. 8:79-88, 2011.

[9] Douet V, Mercier F. 2011. Heparan sulfates in fractones and vascular basement membranes control cell division induced by BMP-7 and FGF-2 in the adult brain. J. Comp. Neurol. In press.

[10] H. A. Eschenauer, N. Olhoff, Topology optimization of continuum structures: A review, Applied Mechanics Reviews 54 (4).

[11] Gordon, M.Y., Riley, GA.P., Watt, S.M., Greaves, M.S. Compartmentalization of a hematopoeitic growth factor (GM-CSF) by glycosamino glycans in the bone marrow microenvironment. Nature. 326A :403-405, 1987.

[12] Hannon, B. and Ruth M. Modeling Dynamic Biological Systems. Springer-Verlag, Series : Modeling Dynamic Systems, New York, 1997

[13] Kerever, A., Schnack, J., Vellinga, D., Ichikawa, N., Moon, C., Arikawa-Hirasawa, E., Efird, J.T., and Mercier, F. Novel extracellular matrix structures in the neural stem cell niche capture the neurogenic factor fibroblast growth factor 2 from the extracellular milieu. Stem Cells 25:2146-2157, 2007.

[14] Leivo I, Vaheri A, Timpl R, Wartiovaara J. Appearance and distribution of collagens and laminin in the early mouse embryo. Dev Biol 76:100-114, 1980.

[15] Leivo I, Wartiovaara J. Basement membrane matrices in mouse embryogenesis, teratocarcinoma differentiation and in neuromuscular maturation. Int J Dev Biol 33:81-89, 1989.

[16] Mercier, F., Kitasako, J.T., and Hatton, G.I.. Anatomy of the brain neurogenic zones revisited: fractones and the fibroblast/macrophage network. J Comp Neurol 451:170-188, 2002.

[17] Mercier, F., Kitasako, J.T., and Hatton, G.I. Fractones and other basal laminae in the hypothalamus. J Comp Neurol 455:324-340, 2003.

[18] Nurcombe V, Fond MD, Wildschut J, Bartlett PF. Developmental regulation of neural response to FGF-1 and FGF-2 by heparan sulfate proteoglycan. Science 260, 103-106, 1993. 
[19] B. Piccoli and M. Garavello Traffic Flow on Network, AMS book series, Applied Math Series n. 1, American Institute of Mathematical Sciences, 2006.

[20] Querin, O. M., Young, V., Steven, G. P., and Xie, Y. M., "Computational efficiency and validation of bi-directional evolutionary structural optimization", Computer methods in applied mechanics and engineering, Vol. 189, No. 2, 2000, pp. 559-573.

[21] Roberts, R., Ghallager, J., Spooncer, E., Allen, T.D., Bloomfield, F., Dexter, T.M. Heparan sulfate-bound growth factor: a mechanism for stromal cell mediated hematopoiesis. Nature. 332:376-378, 1988.

[22] C. . Ronse, M. Tajine. Discretization in Hausdorff space. Journal of Mathematical Imaging and Vision, Vol. 12, no. 3 (2000), pp. 219-242.

[23] G. I. N. Rozvany, Aims, scope, methods, history and unified terminology of computer-aided topology optimization in structural mechanics, Structural and Multidisciplinary Optimization 21.

[24] Sontag E.D.. Some new directions in control theory inspired by systems biology. IET Systems Biology, 1:9-18, 2004.

[25] Sontag, E.D. Molecular systems biology and control. Eur. J. Control, 11(45):396-435, 2005.

[26] Stewart G.R., Pearlman A.L. Fibronectin-like immunoreactivity in the developing cerebral cortex. J Neurosci 7:3325-3333, 1987.

[27] Turing AM. The chemical basis of morphogenesis. Phil Trans R Soc Lond B Biol Sci 237: 37-72, 1952.

[28] Wartiovaara J., Leivo I., Virtanen I., Vaheri A., Graham C.F. (1978). Appearance of fibronectin during differentiation of mouse teratocarcinoma in vitro. Nature 272: 355-356.

[29] Wartiovaara J., Leiva I., Vaheri A. (1979). Expression of the cell surfaceassociated glycoprotein, fibronectin, in the early mouse embryo. Dev. Biol. 69: 247-257.

[30] Yayon, A., Klagsbrun, M., Esko, J.D. Leder, P., Ornitz, D.P. Cell surface, heparin-like molecules are required for binding of basic fibroblast growth factor

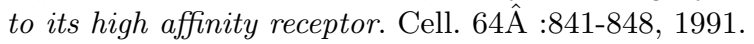

